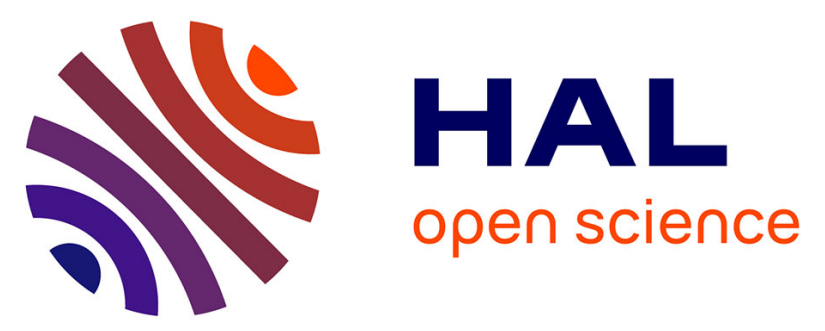

\title{
Experimental evidence of simultaneous multi resonance noise reduction using an absorber with essential non linearity under two excitation frequencies
}

Renaud Côte, Marc Pachebat, Sergio Bellizzi

\section{- To cite this version:}

Renaud Côte, Marc Pachebat, Sergio Bellizzi. Experimental evidence of simultaneous multi resonance noise reduction using an absorber with essential non linearity under two excitation frequencies. Journal of Sound and Vibration, 2014, 333 (20), pp.5057-5076. 10.1016/j.jsv.2014.05.020 . hal-01089548

\section{HAL Id: hal-01089548 \\ https://hal.science/hal-01089548}

Submitted on 2 Dec 2014

HAL is a multi-disciplinary open access archive for the deposit and dissemination of scientific research documents, whether they are published or not. The documents may come from teaching and research institutions in France or abroad, or from public or private research centers.
L'archive ouverte pluridisciplinaire HAL, est destinée au dépôt et à la diffusion de documents scientifiques de niveau recherche, publiés ou non, émanant des établissements d'enseignement et de recherche français ou étrangers, des laboratoires publics ou privés. 


\title{
Experimental evidence of simultaneous multi resonance noise reduction using an absorber with essential non linearity under two excitation frequencies
}

\author{
Renaud Côte $^{\mathrm{a}, *}$, Marc Pachebat ${ }^{\mathrm{a}}$, Sergio Bellizzi ${ }^{\mathrm{a}}$ \\ ${ }^{a}$ LMA, CNRS, UPR 7051, Aix-Marseille Univ, Centrale Marseille, F-13402 Marseille \\ Cedex 20, France
}

\begin{abstract}
The addition of an essentially nonlinear membrane absorber to a linear vibroacoustic system with multiple resonances is studied experimentally, using quasiperiodic excitation. An extended experimental dataset of the system response is analyzed under steady state excitation at two frequencies. Thresholds between low and high damping states within the system and associated noise reduction are observed and quantified thanks to frequency conversion and RMS efficiency indicators. Following previous numerical results, it is shown that the membrane NES (Nonlinear Energy Sink) acts simultaneously and efficiently on two acoustic resonances. In all cases, the introduction of energy at a second excitation frequency appears favorable to lower the frequency conversion threshold and to lower the noise within the system. In particular a simultaneous control of two one-to-one resonances by the NES is observed. Exploration of energy conversion in the two excitation amplitudes plane advocates for a linear dependence of the frequency conversion
\end{abstract}

\footnotetext{
${ }^{*}$ Corresponding author

Email address: cote@lma.cnrs-mrs.fr (Renaud Côte)
} 
thresholds on the two excitation amplitudes.

Keywords: Nonlinear absorber, Nonlinear energy sink, Targeted energy transfer, Quasiperiodic excitation, Acoustic resonance, Noise reduction, Frequency conversion

\section{Introduction}

Targeted Energy Transfer (TET) concept based on an additional essentially nonlinear attachment (also named Nonlinear Energy Sink (NES)) to an existing primary linear system has been extensively studied and it proved to be very efficient for vibration and noise reduction. Various types of forcing have been considered theoretically, numerically and experimentally from impulsive to harmonic and also broadband frequency excitation [1]. The unique nonlinear properties of a NES permit the existence of quasiperiodic responses regimes under periodic excitation which can provide more efficient energy suppression than a linear absorber in the neighborhood of the resonance frequency (one-to-one resonance) [2,3]. A single NES is also able to act on several resonance frequencies of a primary system [4]. All these properties are illustrated experimentally in the context of noise reduction in $[5,6]$.

An interesting question which has been considered more recently concerns the ability of a single NES to provide efficient energy suppression simultaneously on several resonance frequencies of the primary system. The answer is not obvious because there is no validity of the superposition principle due to the intrinsic nonlinear nature of NES. In [7], scenarios of energy transfer between a two degree-of-freedom system and a single NES were investigated numerically under impulsive excitation. The forced case was considered in 
[8] considering external excitation with two additive sinusoidal components at both resonance frequencies of the primary system. It is shown that a two one-to-one resonances of the system is possible simultaneously resulting on vibration reduction around the two resonance frequencies. More complex resonance scenarios are also possible involving inter modulation frequencies. We found consistent results in the acoustic domain, with a system where numerical results are more easily found, and we showed evidences of TET and evidences of strongly modulated regimes [9]. Quasiperiodic forcing have been also considered in [10] but in case of one degree-of-freedom system coupled to a single NES under external excitation with two additive sinusoidal components at frequencies near the resonance frequency of the primary system. It is demonstrated that strong modification of the response regimes can be obtained showing that a single NES can mitigate efficiently the multi-frequency excitation.

The main objective of this study is to obtain experimental confirmations of the simultaneous efficiency of a single NES on a two degree-of-freedom linear system under two-frequency excitation with frequencies near the resonance frequencies of the linear system. We also focus on how the two degreeof-freedom interact with each other and with the NES.

In view of the above objectives, we built a set up in the acoustic domain as in [5] or [11] but now involving several degree-of-freedom. As in [5], a clamped visco-elastic membrane is used as a NES. We checked the classical models and properties on harmonic source experiments, before conducting and analyzing experiments with two-frequency sources. We developed numerical tools for the analysis of complex regimes and voluminous data. 
The paper is organized as follows. In section 2 we describe the experimental setup, then we establish a classical model of our system, and we cheks it with experimental results under harmonic excitation. Section 3 describes the experimental procedure and introduces the original indicators used for the analysis of the results. In Section 4, we analyze in details the responses under two excitation frequencies and we bring some confirmations on theoretical predictions. And finally an overview of the frequency conversion in the two excitation amplitudes plane is proposed.

\section{Experimental set-up and model}

\subsection{Experimental set-up}

The experimental set-up consists in a vibroacoustic system (also named primary system) coupled to a nonlinear absorber (also named nonlinear energy sink or NES). As shown on Fig. 1, the linear primary system is made of two different open pipes, coupled at each end to coupling boxes. One coupling box contains a loudspeaker and a vent, and the nonlinear visco-elastic membrane (the NES) is clamped on one face of the other coupling box.

Three main ideas were taken into account in the design of the set-up. First, the membrane (NES) was designed following the recommendations discussed in [5]. In particular, its material (latex), diameter and thickness were chosen so that the nonlinear behavior can be caused by the pressure amplitude generated inside the coupling box. And a sliding system (visible on Fig. 1 (a)) controlled the pre-stress imposed at the membrane boundaries. Second, on the source side, the parameters of the box and the vent were chosen to ensure a weak coupling between the loudspeaker and the pipes, 
reducing the damping of the pipes by the loudspeaker. And third, on the NES side, the coupling box with NES (Fig. 1 (b)) was chosen sufficiently large to give a weak linear coupling stiffness between the pipes and the membrane.
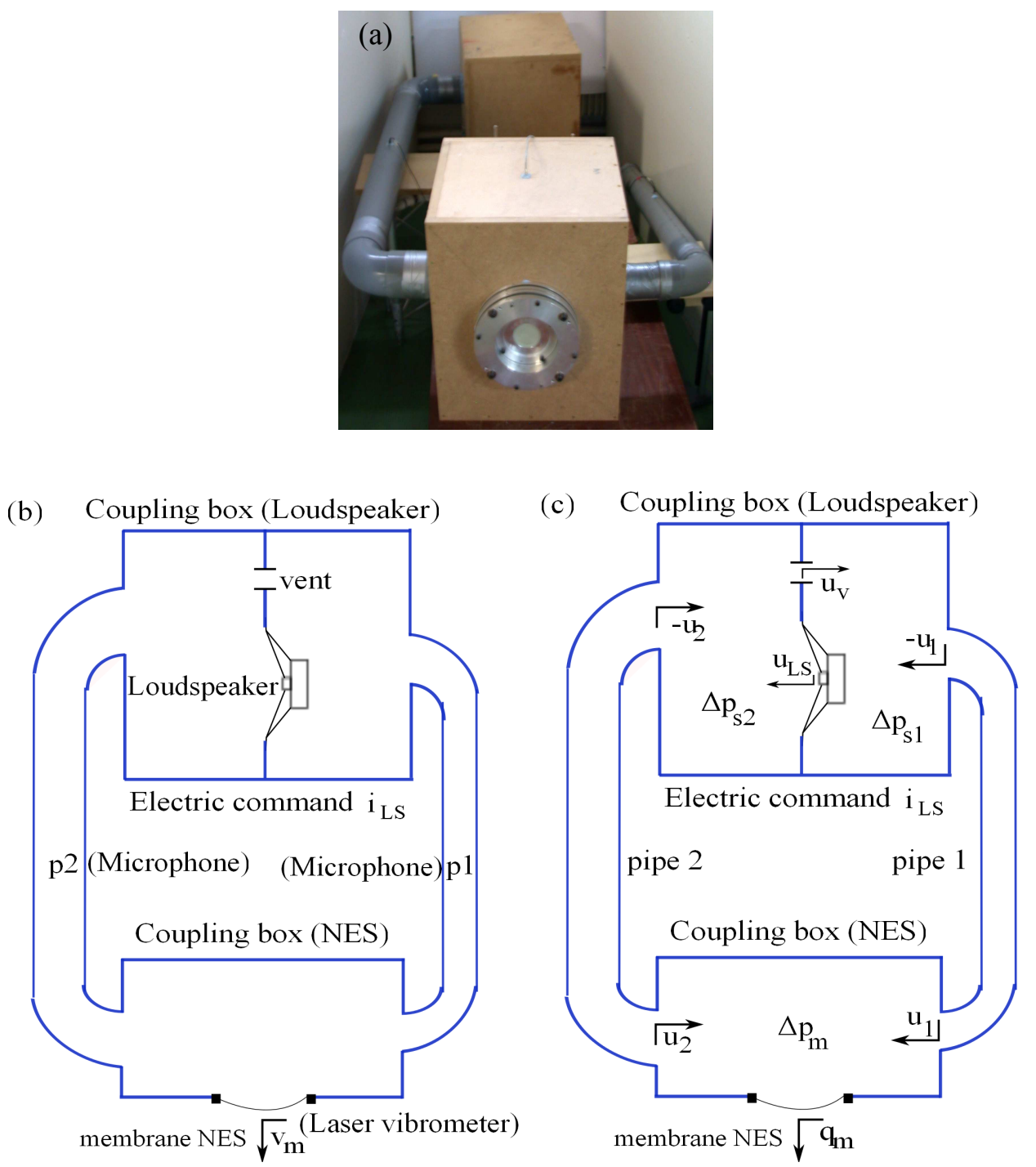

Figure 1: (a) Picture and (b-c) schemas of the set-up. 
During a measurement, a target voltage signal $e(t)$ from a generator (TTI TGA1244) and a power amplifier (TIRA, BAA120), provided an input current signal $i_{\mathrm{LS}}(t)$ to the loudspeaker (current-feedback control). The following responses of the system (see Fig. 1 (b)) were recorded simultaneously (multichannel analyzer/recorder OROS, OR38): the loudspeaker voltage response $e_{\mathrm{LS}}(t)$, the acoustic pressures $p_{1}(t)$ and $p_{2}(t)$ at mid length of the pipes 1 and 2 with two microphones (GRAS, 40BH), and the velocity $v_{\mathrm{m}}(t)$ at the center of the membrane with a laser vibrometer (Polytec, OFV303). The generator target signal $e(t)$ and command signal $i_{\mathrm{LS}}(t)$ were also recorded. Note that the current command $i_{\mathrm{LS}}(t)$ from the amplifier slightly deviates from the target signal $e(t)$, in particular near the resonance frequencies of the primary system. This has been taken into account in the analysis. In order to characterize the linear primary system (see Section 2.3), some measurements were also made with a configuration without NES, by replacing the NES (membrane and the sliding system) by a rigid disk.

The geometrical parameters of the set-up are the following. The dimensions of the membrane (NES) are: $R_{\mathrm{m}}=0.03 \mathrm{~m}$ (radius), $h_{\mathrm{m}}=0.15 \times 0.001 \mathrm{~m}$ (thickness). $S_{\mathrm{m}}$ denotes the associated section area. The volume of the coupling box to NES is: $V_{\mathrm{m}}(=0.35 \times 0.45 \times 0.38)=0.0756 \mathrm{~m}^{3}$. The diameter of the loudspeaker is equal to $0.3 \mathrm{~m}$ giving an effective section area equal to $S_{\mathrm{LS}}\left(=\pi \times 0.129^{2}\right)=0.0523 \mathrm{~m}^{2}$. The dimensions of the vent are: $S_{\mathrm{v}}(=0.38 \times 0.01)=0.0038 \mathrm{~m}^{2}$ (section area), $h_{\mathrm{v}}=0.02 \mathrm{~m}$ (thickness). Each side of the loudspeaker see the same volume $V_{\mathrm{s}_{1}}=V_{\mathrm{s}_{2}}(=0.35 \times 0.45 \times 0.38)=$ $0.0756 \mathrm{~m}^{3}$. The dimensions of pipes 1 and 2 are: $L_{1}=1.81 \mathrm{~m}, L_{2}=1.71 \mathrm{~m}$ (lengths), and $d_{1}=0.072 \mathrm{~m}, d_{2}=0.094 \mathrm{~m}$ (diameter). $S_{1}$ and $S_{1}$ denote the 
associated section area.

\subsection{Associated model}

In this section we present a model of the experimental set-up, based on the assumptions used in [5], [11] and [12] but that includes in addition, a model for the loudspeaker and the vent. The schema and notations of the model are shown on Fig. 1 (c).

Following [5], the nonlinear equation of motion of the membrane (NES) is obtained considering the membrane as a thin elastic structure with geometric nonlinearities and using a 1-DOF Rayleigh-Ritz reduction with a single parabolic shape function to describe the transversal displacement of the membrane. Concerning the linear primary system, four coupled 1-DOF linear equations represent the two pipes, the vent and the loudspeaker. The equations characterizing the pipes are obtained using a 1-DOF Rayleigh-Ritz reduction on the first acoustic mode (with mode shape $\left(\cos \left(\frac{\pi x_{i}}{L_{i}}\right)\right)$ of the wave equation.

The corresponding equations of motion are of the form

$$
\begin{array}{r}
m_{1} \ddot{u}_{1}(t)+2 \tau_{1} \sqrt{k_{1} m_{1}} \dot{u}_{1}(t)+k_{1} u_{1}(t)+S_{1} \Delta p_{\mathrm{m}}(t)+S_{1} \Delta p_{\mathrm{s} 1}(t)=0, \\
m_{2} \ddot{u}_{2}(t)+2 \tau_{2} \sqrt{k_{2} m_{2}} \dot{u}_{2}(t)+k_{2} u_{2}(t)+S_{2} \Delta p_{\mathrm{m}}(t)+S_{2} \Delta p_{\mathrm{s} 2}(t)=0, \\
m_{\mathrm{m}} \ddot{q}_{\mathrm{m}}(t)+k_{\mathrm{m}}\left(\frac{f_{1}^{2}}{f_{0}^{2}} q_{\mathrm{m}}(t)+\eta \dot{q}_{\mathrm{m}}(t)\right)+k_{3}\left(q_{\mathrm{m}}^{3}(t)+2 \eta\left|q_{\mathrm{m}}(t)\right|^{2} \dot{q}_{\mathrm{m}}(t)\right) \\
-\frac{S_{\mathrm{m}}}{2} \Delta p_{\mathrm{m}}(t)=0, \\
m_{\mathrm{v}} \ddot{u}_{\mathrm{v}}(t)+c_{\mathrm{v}} \dot{u}_{\mathrm{v}}(t)+S_{\mathrm{v}} \Delta p_{\mathrm{s} 1}(t)-S_{\mathrm{v}} \Delta p_{\mathrm{s} 2}(t)=0, \\
m_{\mathrm{LS}} \ddot{u}_{\mathrm{LS}}(t)+c_{\mathrm{LS}} \dot{u}_{\mathrm{LS}}(t)+k_{\mathrm{LS}} u_{\mathrm{LS}}(t)+S_{\mathrm{LS}} \Delta p_{\mathrm{s} 1}(t)-S_{\mathrm{LS}} \Delta p_{\mathrm{s} 2}(t)=B l i_{\mathrm{LS}}(t),
\end{array}
$$


where the coupling terms due to the stiffness of the coupling volumes are

$$
\begin{aligned}
& \Delta p_{\mathrm{s} 1}(t)=k_{b_{\mathrm{S} 1}}\left(S_{1} u_{1}(t)+S_{\mathrm{v}} u_{\mathrm{v}}(t)+S_{\mathrm{LS}} u_{\mathrm{LS}}(t)\right), \\
& \Delta p_{\mathrm{s} 2}(t)=k_{b_{\mathrm{S} 2}}\left(S_{2} u_{2}(t)-S_{\mathrm{v}} u_{\mathrm{v}}(t)-S_{\mathrm{LS}} u_{\mathrm{LS}}(t)\right), \\
& \Delta p_{\mathrm{m}}(t)=k_{b_{\mathrm{m}}}\left(S_{1} u_{1}(t)+S_{2} u_{2}(t)-\frac{S_{m}}{2} q_{\mathrm{m}}(t)\right) .
\end{aligned}
$$

Concerning the NES, $q_{\mathrm{m}}$ (Eq. (3)) denotes the displacement of the center of the membrane, and $\dot{q}_{\mathrm{m}}(t)=v_{\mathrm{m}}(t)$ the associated velocity. The parameters satisfy [5]

$$
\begin{gathered}
m_{\mathrm{m}}=\frac{\rho_{\mathrm{m}} S_{\mathrm{m}} h_{\mathrm{m}}}{3}, k_{\mathrm{m}}=\frac{1.015^{4} \pi^{5}}{36} \frac{E h_{\mathrm{m}}^{3}}{\left(1-\nu^{2}\right) R_{\mathrm{m}}^{2}}, \\
f_{0}=\frac{1}{2 \pi} \sqrt{\frac{1.015^{4} \pi^{4} E h_{\mathrm{m}}^{2}}{12\left(1-\nu^{2}\right) \rho_{\mathrm{m}} R_{m}^{4}}} \text { and } k_{3}=\frac{8 \pi E h_{\mathrm{m}}}{3\left(1-\nu^{2}\right) R_{\mathrm{m}}^{2}},
\end{gathered}
$$

where $\rho_{\mathrm{m}}$ (respectively $E, \nu$ and $\eta$ ) denotes the density (respectively Young's modulus, Poisson ratio and viscous damping coefficient) of the membrane. Here $f_{0}$ denotes the first resonance frequency of the membrane without prestress and $f_{1}$ denotes the first resonance frequency of the membrane with prestress in operating conditions. The resonance frequency $f_{1}$ can be measured experimentally so it can be considered as a parameter of the model.

Concerning the linear primary system, $u_{i}(t)$ for $i=1,2$ (Eqs. (1) and (2)) denote the displacement of the air at the end of the pipe $i$ (see Fig. 1 (c)). The acoustic pressure at the middle section of the pipe $i$ is then approximated by

$$
p_{i}(t)=-\frac{\rho_{\mathrm{a}} c_{0} \pi}{L_{i}} u_{i}(t)
$$

where $\rho_{0}$ is the density of the air and $c_{0}$ is the sound wave velocity. The other parameters satisfy

$$
m_{i}=\frac{\rho_{0} S_{i} L_{i}}{2} \text { and } k_{i}=\frac{\rho_{0} c_{0}^{2} \pi^{2} S_{i}}{2 L_{i}} \text { giving } \omega_{i}^{\mathrm{p}^{2}}=\frac{k_{i}}{m_{i}}=\frac{c_{0}^{2} \pi^{2}}{L_{i}^{2}},
$$


where $\omega_{i}^{\mathrm{p}}$ denotes the natural resonance frequency of the acoustic medium inside the open-open pipe $i$, and $\tau_{i}$ denotes the damping ratio of the pipe $i$.

Equation (4) characterizes the motion, $u_{\mathrm{v}}(t)$, of the air inside the vent assuming that the air inside the vent behaves like an ideally rigid solid with mass $m_{\mathrm{v}}=\rho_{0} S_{\mathrm{v}} h_{\mathrm{v}}$. Equation (5) characterizes the motion, $u_{\mathrm{LS}}(t)$, of the diaphragm of the loudspeaker fed with an electrical current $i_{\mathrm{LS}}(t)$, assuming the diaphragm to be rigid (piston). $m_{\mathrm{LS}}$ (respectively $k_{\mathrm{LS}}$ and $c_{\mathrm{LS}}$ ) denotes the mass (respectively stiffness coefficient and damping coefficient). $B l$ denotes the force factor of the loudspeaker (see for example [13]).

Finally, the pressures inside the boxes are assumed spatially uniform and follow the volume variations exerted by the displacements of the vent, the membrane, the loudspeaker diaphragm, and the pipes, as given by Eqs. (6-8) with

$$
k_{b_{\mathrm{s} 1}}=\frac{\rho_{0} c_{0}^{2}}{S_{1}}, k_{b_{\mathrm{s} 2}}=\frac{\rho_{0} c_{0}^{2}}{S_{2}} \text { and } k_{b_{\mathrm{m}}}=\frac{\rho_{0} c_{0}^{2}}{S_{m}} .
$$

For the complete system, substitution of Eqs. (6-8) into Eqs. (1-5), gives the following matrix form system

$$
\mathbf{M} \ddot{\mathbf{U}}(t)+\mathbf{C} \dot{\mathbf{U}}(t)+\mathbf{K U}(t)+\mathbf{F}(\mathbf{U}(t))+\mathbf{G}(\mathbf{U}(t), \dot{\mathbf{U}}(t))=\mathbf{E}(t),
$$

where $\mathbf{U}(t)=\left(u_{1}(t), u_{2}(t), q_{\mathrm{m}}(t), u_{\mathrm{e}}(t), u_{\mathrm{LS}}(t)\right)^{T}$, the matrices $\mathbf{M}, \mathbf{C}$ and $\mathbf{K}$ (not given here) are symmetrical, $\mathbf{F}(\mathbf{U}(t))=\left(0,0, k_{3} q_{\mathrm{m}}^{3}(t), 0,0\right)^{T}, \mathbf{G}(\mathbf{U}(t), \dot{\mathbf{U}}(t))=$ $\left(0,0,2 k_{3} \eta\left|q_{\mathrm{m}}(t)\right|^{2} \dot{q}_{\mathrm{m}}(t), 0,0\right)^{T}$ and $\mathbf{E}(t)=(0,0,0,0, B l)^{T} i_{\mathrm{LS}}(t)$.

\subsection{Linear and nonlinear modes of the system}

Let consider the linear primary system alone (system with a rigid plate instead of the membrane NES). Figure 2 shows the Frequency Response Functions $(\mathrm{FRF}) p_{1} / i_{\mathrm{LS}}$ and $p_{2} / i_{\mathrm{LS}}$ measured using a band limited $([10,200]$ 
$\mathrm{Hz}$ ) white-noise generator signal $e(t)$. Also plotted are the FRF obtained with the linear model defined by the equations of motion (1), (2), (4) and (5) coupled by equations (6-8), with $q_{\mathrm{m}}(t)=0$, and solved algebraically in the frequency domain.

Four resonance peaks appear in the frequency band [20,120] Hz. Roughly speaking, the first resonance (around $35 \mathrm{~Hz}$ ) exhibits a high displacement of the loudspeaker diaphragm whereas the last resonance (around $115 \mathrm{~Hz}$ ) refers mainly to the vent. The two intermediary resonances (around $88 \mathrm{~Hz}$ and 98 $\mathrm{Hz}$ ) exhibit high pressure levels within pipes 1 and 2 (the acoustic medium). Pipe 1 is more sensitive to the resonance around $88 \mathrm{~Hz}$ (note that from Eq. (12), $\omega_{1}^{\mathrm{p}}=88.1 \mathrm{~Hz}$ ) whereas pipe 2 is more sensitive to the resonance around $98 \mathrm{~Hz}\left(\omega_{2}^{\mathrm{p}}=96.09 \mathrm{~Hz}\right)$. But actually, all the four DOF of the primary system are coupled. The coupling boxes, loudspeaker and vent are needed within the model to reproduce accurately the observed FRFs shown on Figure 2. As expected, the design of the setup produces high acoustic pressure levels in both pipes around $88 \mathrm{~Hz}$ and $98 \mathrm{~Hz}$.

A linear modal analysis is also performed on the complete (lossless) linear system with the linear part of the NES (rewritten as Eq. (14)), by using only the matrices $\mathbf{M}$ and $\mathbf{K}$. Results reported in Tab. 1 show that mode 3 and 4 exhibit high modal amplitudes $u_{1}$ and $u_{2}$ within the pipes (the acoustic medium). Mode number 3 exhibits out-of-phase displacements within to the two pipes $\left(u_{1}\right.$ and $u_{2}$ have opposite sign) whereas mode number 4 exhibits in-phase displacements. This behavior indicates that the membrane do not interact symmetrically on the two acoustic modes number 3 and 4 .

Let now consider the complete (nonlinear) system under sinusoidal excita- 

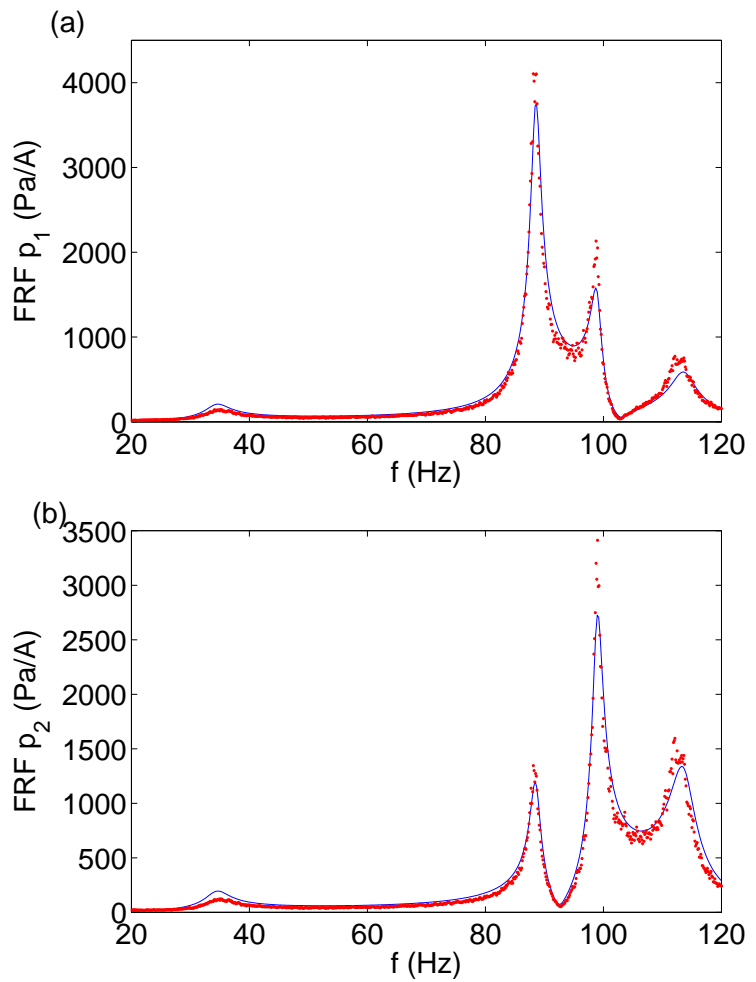

Figure 2: FRF (a) $p_{1} / i_{\mathrm{LS}}$ (b) $p_{2} / i_{\mathrm{LS}}$, system without NES. Comparison experiment (dotted lines) and model (continuous line).

tion $e(t)=E \cos (2 \pi f t)$. Figure 3 shows the RMS values of the steady-state responses $p_{1}, p_{2}$ and $v_{m}$. The excitation frequency $f$ was chosen from 85 $\mathrm{Hz}$ to $104 \mathrm{~Hz}$ with step $0.1979 \mathrm{~Hz}$, including the two resonance frequencies of modes 3 and 4 . Five excitation levels were used: $E=0.004375 \mathrm{~V}$, $E=0.07 \mathrm{~V}, E=0.07875 \mathrm{~V}, E=0.1312 \mathrm{~V}$ and $E=0.2275 \mathrm{~V}$. Also plotted are the responses obtained with the complete nonlinear model defined by equations of motion (14by using the ordinary differential equations solver NDSolve (with the choice Automatic for the option Method) available 
in (C)Mathematica.
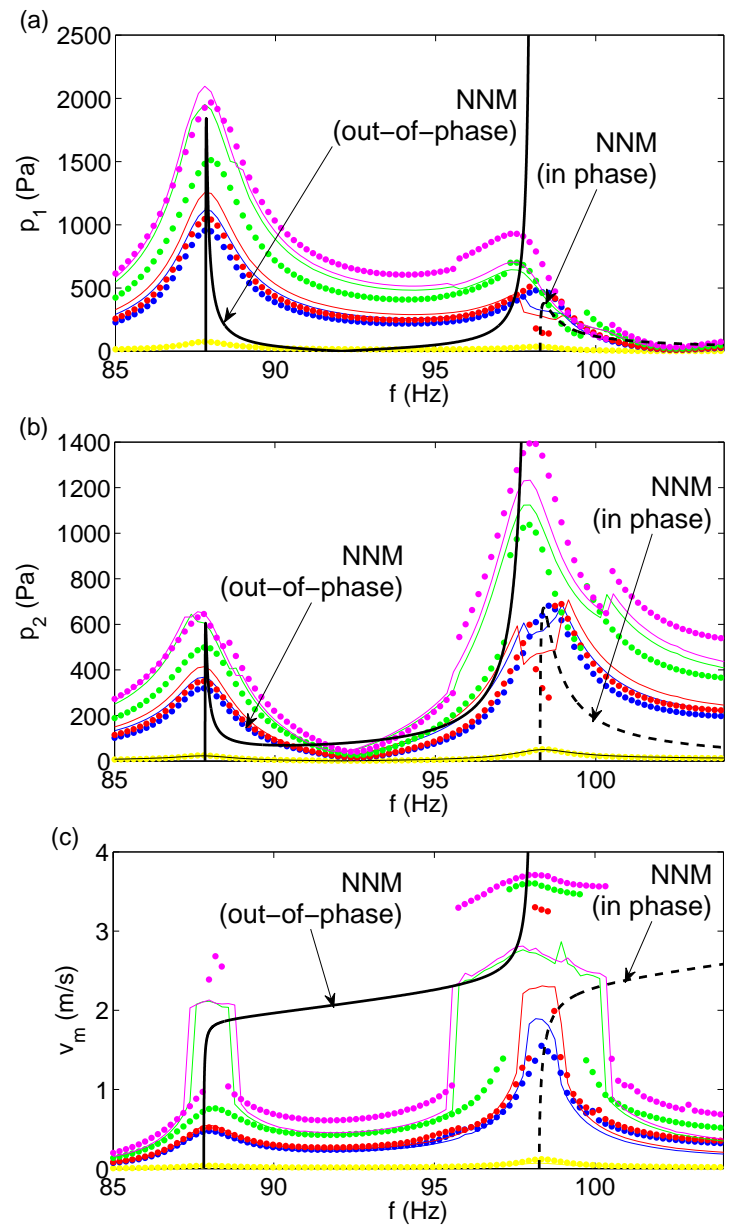

Figure 3: RMS responses (a) $p_{1}$, (b) $p_{2}$ and (c) $v_{\mathrm{m}}$, system with NES. Comparison of experiment (dotted lines) and model (continuous lines) for several excitation levels: $E=0.004375 \mathrm{~V}$ (yellow curves), $E=0.07 \mathrm{~V}$ (blue curves), $E=0.07875 \mathrm{~V}$ (red curves), $E=0.1312 \mathrm{~V}$ (green curves) and $E=0.2275 \mathrm{~V}$ (magenta curves); also shown: Nonlinear Normal Modes (black curves).

At low excitation level $(E=0.004375 \mathrm{~V})$, the model responses are in very good agreement with data (Fig. 3, yellow curves)). Increasing the level 


\begin{tabular}{lccccc}
\hline Mode number & 1 & 2 & 3 & 4 & 5 \\
\hline$f_{i}(\mathrm{~Hz})$ & 29.57 & 34.45 & 87.83 & 98.32 & 113.52 \\
$u_{1}$ & -0.0093 & 0.0064 & 1.0000 & -0.5182 & 0.0738 \\
$u_{2}$ & -0.0084 & -0.0051 & -0.2993 & -0.8485 & -0.1463 \\
$q_{\mathrm{m}}$ & -1.0000 & 0.0211 & -0.2130 & 0.6036 & 0.0379 \\
$u_{\mathrm{v}}$ & -0.0063 & -1.0000 & -0.8965 & -1.0000 & 1.0000 \\
$u_{\mathrm{LS}}$ & 0.002 & 0.0570 & -0.0837 & -0.0851 & 0.0783 \\
\hline
\end{tabular}

Table 1: Resonance frequencies and mode shapes of the undamped underlying linear model with membrane NES.

( $E=0.07 \mathrm{~V}$ and $E=0.07875 \mathrm{~V})$, the model is able to reproduce the nonlinear behaviors (for example, the response level decreases when the excitation level increases, steps appear on the membrane response). These behaviors are first observed around the resonance frequency $98.3 \mathrm{~Hz}$ (Fig. 3, blue and red curves). Still increasing the level $(E=0.1312 \mathrm{~V}$ and $E=0.2275 \mathrm{~V})$, some nonlinear behaviors are also visible around the resonance frequency $88 \mathrm{~Hz}$ (Fig. 3, green and magenta curves). In the considered excitation range, the model gives responses which are in good agreement with measurements for the acoustic pressures $p_{1}$ and $p_{2}$. However, the model is not able to reproduce well the response level of the membrane in particular at high excitation level. The upper bounds observed on $v_{\mathrm{m}}$ at high excitation levels are underestimated by the model. This is probably due to the membrane model which cannot reproduce the complex motion of the membrane.

Also reported in Fig. 3 are the Nonlinear Normal Modes (NNMs) associated to the out-of-phase and in-phase acoustic modes. The free software 
ManLab [14] was used to compute the NNMs as families of periodic solutions of the autonomous undamped associated mechanical system. It is based on the harmonic balance method (HBM) [15] and the so-called asymptotic numerical method [16]. In HBM, there is a choice of the order of approximation based on the hypothesis that higher frequencies in the reponses do not change significantly the solutions. We checked that the fifth order was enough: using higher orders of approximation did not improve the results.

As described in [17], the NNMs can be used to estimate the forcing level for the beginning of TET. This threshold is defined as the forcing level where the resonance peak of the periodic response coincides with the maximum point of the second nonlinear mode around the considered mode. For the in-phase mode, the threshold is near $E_{\mathrm{TET}} \approx 0.07$ (see Fig. 3(b), blue curve) whereas the threshold is near $E_{\mathrm{TET}} \approx 0.2275$ for the out-of-phase mode (see Fig. 3(b), magenta curve). These values are significantly different and confirm that the membrane acts preferentially on the in-phase NNM. This point will be considered in the following discussions.

Results presented above share the following set of parameter values. For the membrane (NES), the numerical values of the material parameter (latex) are: $\rho_{\mathrm{m}}=980 \mathrm{~kg} \mathrm{~m}^{-3}, E=1480000 \mathrm{~Pa}$ and $\nu=0.49$. Parameters $f_{1}$ and $\eta$ (Eq. (3)) were chosen to $f_{1}=4.5 \mathrm{~Hz}$ and $\eta=0.0006$. Note that the value $f_{1}$ is close the $f_{0}$ value (equal here to $3.75 \mathrm{~Hz}$ ) which is in agreement with the sliding system adjustment of the membrane pre-stress. For the primary linear system, the numerical values for the damping parameters were chosen as: $\tau_{1}=\tau_{2}=0.007, c_{\mathrm{v}}=0.0031 \mathrm{Ns} / \mathrm{m}, c_{\mathrm{LS}}=3.5186 \mathrm{Ns} / \mathrm{m}$. We adjusted $\eta, \tau_{1}, c_{\mathrm{v}}$ and $c_{\mathrm{LS}}$ empirically to get the best fit of the model to experimental data. Our 
previous works showed that these values are of the same order of magnitude as the usual physical values. The geometrical parameter values used within the models are exactly the physical values of the set-up (see Section 2.1), except for the following: the lengths of the pipes $\left(L_{1}=1.81+0.1195 \mathrm{~m}\right.$, $\left.L_{2}=1.71+0.059 \mathrm{~m}\right)$ and the mass of the vent $\left(m_{\mathrm{v}}=1.5288 \times 10^{-4} \times 3.25 \mathrm{~kg}\right)$. These corrections can be seen as a simplified way for the model (Eq. (14)) to include other phenomena that exist in experimental set-up, that is, the added lengths at geometrical discontinuities and added mass of the loudspeaker due to its radiation impedance. The loudspeaker parameters values were taken from the manufacturer specifications: $m_{\mathrm{LS}}=0.07 \mathrm{~kg}$., $k_{\mathrm{LS}}=6908.7 \mathrm{~N} / \mathrm{m}$ and $B l=10.7 \mathrm{~N} / \mathrm{A}$. The parameter values for air are: $\rho_{0}=1.2 \mathrm{~kg} \mathrm{~m}^{-3}$, $c_{0}=340 \mathrm{~m} \mathrm{~s}^{-1}$.

\section{Test procedure and analysis tools under two excitation frequen- cies}

\subsection{Two excitation frequency tests}

The input current command signal $i_{\mathrm{LS}}(t)$ into the loudspeaker is driven from the generator by a voltage signal of the form

$$
e(t)=A \cos \left(2 \pi f^{A} t\right)+B \cos \left(2 \pi f^{B} t+\phi\right)
$$

where $f^{A}$ (respectively $f^{B}$ ) denotes the excitation frequency near the resonance frequency of the out-of-phase (respectively in-phase) acoustic mode and $A$ (respectively $B$ ) is the associated excitation amplitude. The phase $\phi$ is introduced arbitrarily by the signal generator.

A measurement run consists in making a series of experiments, where the value of the scanning frequency $f^{A}$ (respectively $f^{B}$ ) is updated for each 
experiment, while the three other parameters $f^{B}, A$ and $B$ (respectively $f^{A}$, $A$ and $B$ ) remain unchanged. The hysteresis properties (or occurrence of multi-solutions) are not considered in this experimental investigation. Each experiment is done with constant values for $A, B, f^{A}$ and $f^{B}$. The duration of an experiment must be limited for practical reasons, but must be long enough to capture the physics of the reponse. We have chosen a duration of 13 seconds for each experiment. There are 2 steps in an experiment. The first step lasts 3 seconds with no source signal. It permits to get null initial conditions, whatever happened before. The second step lasts 10 seconds with the source on, but we record only the last 7 seconds, the first 3 seconds permiting to vanish the transitory effects of excitation. For a source signal composed of multi-frequency components (in our case at least, $f^{A}$ and $f^{B}$ ) the recording duration, has to be large with respect to the greater period of the inter modulation terms. In this study, the recording duration of $7 \mathrm{~s}$, is more than 30 times longer than the longest beating period of the source $\left(7>30 \operatorname{Max}\left(1 /\left|f^{A}-f^{B}\right|\right)\right)$.

A measurement test consists in making a series of runs where the value of the amplitude $A$ (resp. $B$ ) is updated for each run. Nine tests were performed, gathering a total of 19123 experiments constituing 299 runs. They are presented in Table 2. The frequency step $\delta f=0.1979 \mathrm{~Hz}$ was used to define runs in bands $\left[f_{\min }^{A}, f_{\max }^{A}\right]$ and $\left[f_{\text {min }}^{B}, f_{\text {max }}^{B}\right]$. Steps in amplitude bands $\left[A_{\min }, A_{\max }\right]$ and $\left[B_{\min }, B_{\max }\right]$ are equal to $0.00875 \mathrm{~V}$ in case of large amplitude interval (Tests 1, 5, 6, 8) and equal to $0.004375 \mathrm{~V}$ for the other cases.

Three types of tests are encountered. The first type of test (Tests 1 and 


\begin{tabular}{ccccc}
\hline & $A$ & $B$ & $f^{A}$ & $f^{B}$ \\
Test & {$\left[A_{\text {min }}, A_{\text {max }}\right]$} & {$\left[B_{\text {min }}, B_{\text {max }}\right]$} & {$\left[f_{\text {min }}^{A}, f_{\text {max }}^{A}\right]$} & {$\left[f_{\text {min }}^{B}, f_{\text {max }}^{B}\right]$} \\
& $(\mathrm{V})$ & $(\mathrm{V})$ & $(\mathrm{Hz})$ & $(\mathrm{Hz})$ \\
\hline 1 & 0 & {$[0.004375,0.35]$} & - & {$[94 ., 104]$.} \\
2 & $2 . \times B$ & {$[0.004375,0.09]$} & 88. & {$[94 ., 104]$.} \\
3 & $4 . \times B$ & {$[0.004375,0.045]$} & 88. & {$[94 ., 104]$.} \\
4 & $8 . \times B$ & {$[0.004375,0.02]$} & 88. & {$[94 ., 104]$.} \\
5 & {$[0.004375,0.35]$} & 0.066 & 88. & {$[94 ., 104]$.} \\
6 & {$[0.004375,0.35]$} & 0 & {$[85 ., 95]$.} & - \\
7 & {$[0.004375,0.175]$} & $A / 2$. & {$[85 ., 95]$.} & 98.8 \\
8 & {$[0.004375,0.35]$} & $A / 8$. & {$[85 ., 95]$.} & 98.8 \\
9 & 0.175 & {$[0.0,0.09]$} & {$[85 ., 95]$.} & 98.8 \\
\hline
\end{tabular}

Table 2: Excitation parameters for the tests.

6) corresponds to (single frequency) sinusoidal excitation and will be used as reference and compared with the existing results $[4,5]$. The second type of test (Tests 2,3,4 and 7,8) exhibits a second (fixed) excitation frequency in addition to the scanning frequency, with constant amplitude ratio $A / B$ in $e(t)$. For the third type of test (Tests 5 and 9), the second (fixed) excitation frequency explore different parameter amplitudes in $e(t)$, while the amplitude of the scanning frequency is kept constant. The sampling frequency $f_{s}=$ $4096 \mathrm{~Hz}$ is well above the source frequencies and the higher harmonics used in the model. 


\subsection{Signal analysis under two excitation frequencies}

\subsubsection{Classical RMS analysis}

The RMS values is used to characterize the steady state responses of the system. For a recorded (at the sampling frequency $f_{\mathrm{s}}$ ) signal $s\left(t_{n}\right)$ of length $N$ (here $N=7 f_{s}=28672$ ), the RMS value of $s$ is defined as

$$
s_{\mathrm{RMS}}=\sqrt{\frac{1}{N} \sum_{n=0}^{N}\left(s\left(t_{n}\right)-\bar{s}\right)^{2}}
$$

where $\bar{s}$ denotes the arithmetic mean of $s\left(t_{n}\right)$.

\subsubsection{Defining the energy frequency conversion ratios $H C R$ and $N H C R$}

For nonlinear systems under two excitation frequencies, $f^{A}$ and $f^{B}$, the frequency distribution of energy of the response signals (here the magnitude of the Fourier spectrum) may be divided into three parts. The first part is the energy at frequencies identical to the excitation frequencies $f^{A}$ and $f^{B}$. The second part is the energy localized at inter modulation frequencies of $f^{A}$ and $f^{B}$, defined as linear combinations of $f^{A}$ and $f^{B}$ (except $f^{A}$ and $f^{B}$ ). This energy is used to define a criterion called here the harmonic conversion ratio $(\mathrm{HCR})$. And the third part is the energy localized at whatever frequency different from $f^{A}, f^{B}$ and any of their possible inter modulation frequencies. This energy is used to define a criteria called here the nonharmonic conversion ratio $(\mathrm{NHCR})$.

Let focus now on an estimation procedure for the two indicators HCR and NHCR. Since the excitation frequencies $f^{A}$ and $f^{B}$ do not coincide with frequencies of the Fourier analysis, the problem is to subtract from the Fourier spectrum, the correct peak shape and magnitude at a set of known frequencies. A quite accurate approach to this problem is discussed in [18]. We 
propose here a simplified procedure assuming well separated peaks in the Fourier space, which is generally the case in our study for the most important contributions. As explained below, peak shape and magnitude to be subtracted at a given frequency is based on the Fourier spectrum of a windowed cosine function at that frequency.

For a recorded (at the sampling frequency $f_{\mathrm{s}}$ ) signal $s\left(t_{n}\right)$ of length $N$ (here $N=28672$ ), the discrete Fourier transform combined with Hamming function windowing defines the following Fourier spectrum

$$
F S(s, k)=S(k)=\sum_{n=0}^{N-1} s\left(t_{n}\right)\left(0.54-0.46 \cos \left(\frac{2 \pi n}{N-1}\right)\right) e^{j 2 \pi k \frac{n}{N}}
$$

at the discrete analysis frequencies $k \Delta f_{\mathrm{s}}$, with $\Delta f_{s}=\frac{f_{\mathrm{S}}}{N}$ and $j^{2}=-1$. Let $K$ be the cutoff order of inter modulation frequencies. We denote $H_{K}$ the ensemble of $f^{A}, f^{B}$, and the inter modulation frequencies up to order $K$, defined as

$$
H_{K}=\left\{\left|\alpha f^{A}+\beta f^{B}\right| \text { such that }(\alpha, \beta) \in \mathbb{Z}^{2}, 0<|\alpha|+|\beta| \leq K\right\} .
$$

$H_{K}$ is a discrete set of frequencies, $M=\operatorname{card}\left(H_{K}\right)$ is the number of elements of $H_{K}$. The elements of $H_{K}$ are labeled along their computation order: $H_{K}=\left\{f^{1}, f^{2}, \cdots, f^{M}\right\}$, with $f^{1}=f^{A}$, and $f^{2}=f^{B}$. For a given $f^{l} \in H_{k}$, we define the nearest discrete Fourier analysis frequency by the integer

$$
k_{l}=R\left(\frac{f^{l}}{\Delta f_{\mathrm{s}}}\right)
$$

where $R(x)$ denotes the nearest integer function of real number $x$.

The harmonic conversion ratio (HCR) of the energy of the signal $s$ is defined as

$$
\operatorname{HCR}(s)=\frac{\sum_{k=0}^{N-1}\left(D_{M}(k)-D_{2}(k)\right)^{2}}{\sum_{k=0}^{N-1}|S(k)|^{2}}
$$


where, for each $k$, the sequence $D_{1}(k), D_{2}(k), \cdots, D_{M}(k)$ is defined by the recursive formula

$$
\left\{\begin{array}{c}
D_{0}(k)=|S(k)| \\
D_{l}(k)=\operatorname{Max}\left(0,\left(D_{l-1}(k)-\left|\frac{C_{l}(k)}{C_{l}\left(k_{l}\right)}\right| D_{l-1}\left(k_{l}\right)\right)\right) . \\
\quad \text { for } l=1, \cdots M
\end{array}\right.
$$

In Eq. 21, $C_{l}(k)$ denotes the Fourier spectrum of the time function $c_{l}(t)=$ $\cos \left(2 \pi f^{l} t\right)$ as defined by Eq. (17)

$$
C_{l}(k)=F S\left(c_{l}, k\right) .
$$

Note that for the particular case of (single frequency) sinusoidal excitation ( $f^{A}$ or $f^{B}$ equal to zero), the set $H_{K}$ (Eq. 18) corresponds to purely harmonic frequencies (multiples) of the single excitation frequency.

To analyze the distribution of the frequency components which are not inter modulation products of $f^{A}$ and $f^{B}$, we introduce the nonharmonic conversion ratio (NHCR) of the energy of the signal $s$ as

$$
\operatorname{NHCR}(s)=1-\frac{\sum_{k=0}^{N-1} D_{M}(k)^{2}}{\sum_{k=0}^{N-1}|S(k)|^{2}} .
$$

Note that $\operatorname{HCR}(s)+\operatorname{NHCR}(s)$ is not equal to one. The complement to one corresponds to the fraction of energy at the excitation frequencies $f^{A}$ and $f^{B}$.

For a linear system, we have $\operatorname{HCR}(s)=\operatorname{NHCR}(s)=0$ which characterizes signals with energy only localized on the excitation frequencies $f^{A}$ and $f^{B}$. The combination $\operatorname{HCR}(s)$ large with $\operatorname{NHCR}(s)=0$ is associated to signals with energy localized on the inter modulation frequencies of $f^{A}$ and $f^{B}$. It corresponds, for example, to a classical high frequency enrichment 
in nonlinear systems. The combination $\operatorname{HCR}(s)=0$ with $\operatorname{NHCR}(s)$ large is associated to signals with energy localized on the excitation frequencies $f^{A}$ and $f^{B}$ plus other frequencies non commensurable with $f^{A}$ and $f^{B}$. This combination can be used to detect a weakly modulated response appearing in the vicinity of Hopf bifurcation. Finally the combination $\operatorname{HCR}(s)$ large and $\operatorname{NHCR}(s)$ large can correspond to strongly modulated responses or more generally to the transition to chaos

The ratios HCR and NHCR are only computed for the membrane velocity $v_{\mathrm{m}}$, the most sensitive measured response to nonlinear effects.

\subsubsection{Efficiency RMS ratio compared to the underlying linear system}

For the evaluation of the efficiency of the membrane NES on the primary system, we built another indicator based on the comparison of the actual system response with the corresponding system response assuming linear behavior (also named underlying linear system). These linear responses which could not be measured, are estimated on the basis of the response of the nonlinear system at low excitation level.

For a given signal with two frequencies from the generator

$$
e(t)=A \cos \left(2 \pi f^{A} t\right)+B \cos \left(2 \pi f^{B} t+\phi\right)
$$

with amplitude $(A, B)$, we introduce the efficiency RMS ratio on the pipe $i$ for $i=1,2$ as

$$
\frac{p_{i_{\mathrm{RMS}}}}{p_{i_{\mathrm{RMS}}}^{\mathrm{L}}}
$$

where $p_{i_{\mathrm{RMS}}}$ denotes the RMS value of the sound pressure $p_{i}$ measured under (24) and $p_{i_{\text {RMS }}^{\mathrm{L}}}^{\mathrm{L}}$ the RMS value of the sound pressure considering the 
underlying linear system. $p_{i_{\mathrm{RMS}}}^{\mathrm{L}}$ is estimated by

$$
\left(p_{i_{\mathrm{RMS}}}^{\mathrm{L}}\right)^{2}=\rho_{A} p_{i}\left(A_{m i n}, 0\right)_{\mathrm{RMS}}^{2}+\rho_{B} p_{i}\left(0, B_{m i n}\right)_{\mathrm{RMS}}^{2}
$$

where $p_{i}\left(A_{\text {min }}, 0\right)_{\mathrm{RMS}}$ (respectively $\left.p_{i}\left(0, B_{\text {min }}\right)_{\mathrm{RMS}}\right)$ denotes the RMS value of the sound pressure measured at low level under sinusoidal signal $e(t)=$ $A_{\text {min }} \cos \left(2 \pi f^{A} t\right)$ (respectively $\left.e(t)=B_{\text {min }} \cos \left(2 \pi f^{B} t\right)\right)$ during Test 1 (resp. Test 6), levels for which the responses of the system are assumed to be linear. $A_{\text {min }}$ and $B_{\text {min }}$ are defined in Tab. 2. The weighting coefficients $\rho_{A}$ and $\rho_{B}$ are defined as a ratio between the Fourier components (as defined in Eq. 17) of the current $i_{\mathrm{LS}}$ measured under $(24)$, and $i_{\mathrm{LS}}\left(A_{\text {min }}, 0\right)$ and $i_{\mathrm{LS}}\left(0, B_{\text {min }}\right)$ measured at low level under sinusoidal signals i.e

$$
\rho_{A}=\frac{I_{\mathrm{LS}}\left(k_{A}\right)}{I_{\mathrm{LS}}\left(A_{\min }, 0\right)\left(k_{A}\right)} \text { and } \rho_{B}=\frac{I_{\mathrm{LS}}\left(k_{B}\right)}{I_{\mathrm{LS}}\left(0, B_{\min }\right)\left(k_{B}\right)} \text {. }
$$

The weighting coefficients $\rho_{A}$ and $\rho_{B}$ are evaluated at the nearest Fourier analysis frequencies from $f^{A}$ and $f^{B}$ respectively, namely at $k_{A}=R\left(f^{A}\right)$ and $k_{B}=R\left(f^{A}\right)$. The additive rule $(26)$ holds because $p_{i}\left(A_{\min }, 0\right)$ and $p_{i}\left(0, B_{\min }\right)$ are orthogonal since $f^{A} \neq f^{B}$. As long as the behavior of the system is linear, the efficiency RMS ratios are equal to one. A ratio value smaller than one indicates that the non linearity acts as an absorber of noise whereas a ratio value greater than one indicates a degradation of the performance of the system.

\subsubsection{Fourier magnitude ratio at excitation frequencies}

Finally, more classical is to consider the Fourier magnitude ratios between the Fourier components of the sound pressure $p_{i}$ for $i=1,2$ and the current $i_{\mathrm{LS}}$

$$
\frac{P_{i}\left(k_{A}\right)}{I_{\mathrm{LS}}\left(k_{A}\right)} \text { and } \frac{P_{i}\left(k_{B}\right)}{I_{\mathrm{LS}}\left(k_{B}\right)}
$$


evaluated at the nearest Fourier analysis frequencies from $f^{A}$ and $f^{B}$ respectively, namely at $k_{A}=R\left(f^{A}\right)$ and $k_{B}=R\left(f^{A}\right)$.

These ratios indicate the relative responses in the tubes as a function of the excitation frequencies. When the excitation frequencies are close to the resonance frequencies, they are related to the amplitude of the resonant motion components.

\section{Results and discussion}

Among the complete data set described in Table 2, for sake of clarity, only Tests 1 and 6 (single sinusoidal excitation) and Tests 2, 5 and 8 (two excitation frequencies) are considered here for a detailed presentation and discussion. For each test, 12 selected runs are showed for readability. For each test, two types of figure are proposed to analyze the results.

The first type of figure (called Board 1) includes eight plots showing (a) the RMS values of the command $i_{\mathrm{LS}}$, (b) the RMS value of the membrane NES velocity $v_{\mathrm{m}}$, (c,d) the energy frequency conversion ratios HCR and NHCR computed from $v_{\mathrm{m}},(\mathrm{e}, \mathrm{f})$ the RMS values of the sound pressures

$p_{1}$ and $p_{2}$ and $(\mathrm{g}, \mathrm{h})$ the efficiency RMS ratio for $p_{1}$ and $p_{2}$. For a given excitation level, each quantity is plotted as a continuous curve versus scan frequencies.

The second type of figure (called Board 2) is used only for tests with two excitation frequencies (Tests 2,5 and 8). It includes four plots showing $(\mathrm{a}, \mathrm{b})$ the Fourier magnitude ratios of the sound pressure $p_{1},(\mathrm{c}, \mathrm{d})$ the Fourier magnitude ratios of the sound pressure $p_{2}$. For a given excitation level (a run), each quantity is plotted as a continuous curve versus scan frequencies. 
Finally, to easily localize the nonlinear domains resulting from the analysis of the energy frequency conversion ratios $\operatorname{HCR}\left(v_{\mathrm{m}}\right)$ and $\operatorname{NHCR}\left(v_{\mathrm{m}}\right)$, the four following conventions have been adopted during the plots: (i) a circle marker is added at all the results of the run if for this $\operatorname{run} \operatorname{HCR}\left(v_{\mathrm{m}}\right) \geq$ 0.02, (ii) a star marker is added at all the results of the run if for this run $\operatorname{NHCR}\left(v_{\mathrm{m}}\right) \geq 0.02$, (iii) on each plot, the curve corresponding to the lowest excitation level where at least in one experiment $\operatorname{HCR}\left(v_{\mathrm{m}}\right)$ exceeds 0.02 , is indicated by thick line, the corresponding excitation level is denoted $\left(A_{\mathrm{HCR}}, B_{\mathrm{HCR}}\right)$ (and named HCR threshold) and (iv) on each plot, the curve corresponding to the lowest excitation level where at least in one experiment $\operatorname{NHCR}\left(v_{\mathrm{m}}\right)$ exceeds 0.02 , is indicated by thick line, the corresponding excitation level is denoted $\left(A_{\mathrm{NHCR}}, B_{\mathrm{NHCR}}\right)$ (and named NHCR threshold).

For each test, the 12 excitation levels have been selected uniformly between the low and high excitation levels and including the best estimated levels for $\left(A_{\mathrm{HCR}}, B_{\mathrm{HCR}}\right)$ and $\left(A_{\mathrm{NHCR}}, B_{\mathrm{NHCR}}\right)$ at the scale of the amplitude step used during the test.

\subsection{Tests 1 and 6: single frequency excitation}

The behavior of the system under (single frequency) sinusoidal excitation, scanned around the in-phase mode (Test 1 in Table 2), is presented on Fig. 4 (Board 1).

Concerning the loudspeaker command level, the power amplifier driving the loudspeaker in current-feedback mode provides a nearly constant command $i_{\text {LS }}$ (see Fig. 4(a)), for the 12 runs (lines) presented. Concerning the system response, as expected, a resonance peak around the in-phase mode (mode $4, f_{4}=98.32 \mathrm{~Hz}$, in Table 1 ) is observed on the sound pressures 
responses within the pipes (Fig. 4(e,f)) for low excitation levels. At the excitation level $B_{\mathrm{HCR}}=0.07875$, the run exhibits sudden abrupt changes in the system response. These "discontinuities" correspond to jumps of the membrane velocity $\mathrm{RMS}$ values between low $(\approx 1.2 \mathrm{~m} / \mathrm{s})$ and high $(\approx 3.2$ $\mathrm{m} / \mathrm{s}$ ) values (see Fig. 4(b)) associated to a significant reduction of noise in both pipes (see Fig. $4(\mathrm{e}, \mathrm{f})$ ). The $B_{\mathrm{HCR}}$ value is near the TET threshold $\left(E_{\mathrm{TET}}=0.07\right)$ predicted by the model (see Section 2.3$)$. These discontinuities correspond to a state change of the nonlinear system as described in [4]. The membrane NES efficiency is at its best when $B$ is greater or equal to $B_{\mathrm{HCR}}=0.07875$ but not too high: the responses within pipes represent only $30 \%$ of the underlying linear system responses at $B=B_{\mathrm{HCR}}$ and this percent slightly increases when $B$ increases (Fig. $4(\mathrm{~g}, \mathrm{~h})$ ). In all these cases, the RMS values of $v_{m}$ is greater than $3 \mathrm{~m} / \mathrm{s}$ (see Fig. $4(\mathrm{~b})$ ).

Fig. 4(h) shows values above 1 . for the efficiency RMS ratios for $p_{2}$, meaning that the non linearity increases the response within the pipes. It is due to the left shift of the in-phase mode resonance at high excitation levels, a common feature in such nonlinear systems. From an efficiency point of view, values above 1 . observed here are not critical since they occur at low response amplitude far from the in-phase mode resonance.

In addition to expected results, the analysis of the energy frequency conversion ratios proposed in Section 3.2 can give new insight into the nature and intensity of nonlinear phenomena. On one side, Fig. 4(d) shows that the harmonic conversion ratios $\operatorname{HCR}\left(v_{\mathrm{m}}\right)$ never exceed 6\%. Only a small amount of the energy is localized into high order harmonics of the excitation frequency $f^{B}$ (remember that $H_{k}$ (Eq. (18)) is here reduced to multiples 
of $f^{B}$ ). It confirms that approximated solutions from models with limited number of harmonics (as in Eq. (3) and for example in [4], [7] and [9]) are adequate to describe the membrane NES motion. But on the other side, on Fig. $4(\mathrm{c}), \operatorname{NHCR}\left(v_{\mathrm{m}}\right)$ can easily exceed $20 \%$, even for low excitation level $B$. Fourier transforms of $v_{m}$ (not shown) reveal that nonharmonic conversion results in frequencies greater than (but not multiple of) $f^{B}$, that are more easily damped.

The energy frequency conversion ratios $\operatorname{HCR}\left(v_{\mathrm{m}}\right)$ and $\operatorname{NHCR}\left(v_{\mathrm{m}}\right)$ seem adapted to detect jumps changes in the nonlinear system behavior. First because, frequencies with high $\operatorname{HCR}\left(v_{\mathrm{m}}\right)(\geq 0.02)$ on Fig. 4(d) clearly coincide with frequencies exhibiting high RMS values of $v_{m}$ on Fig. 4(b), where the membrane motion exhibits a saturation behavior due to its stiffening non linearity. And second because, high $\operatorname{NHCR}\left(v_{\mathrm{m}}\right)(>0.02)$ combined with small $\operatorname{HCR}\left(v_{\mathrm{m}}\right)(<0.02)$ on Fig. 4(c) always appears at the immediate vicinity of frequencies where $v_{m}$ is low $\left(v_{m} \approx 1 \mathrm{~m} / \mathrm{s}\right)$ but close to jumps from low to high RMS values on Fig. 4(b). Fourier transforms of $v_{m}$ (not shown) reveal the presence of satellite peaks (outside the harmonics of the excitation frequency) around the main peak at the excitation frequency. This scenario is compatible with the neighborhood of Hopf bifurcation as observed numerically for a similar system in [19].

Finally, Test 1 also exhibits high $\operatorname{NHCR}\left(v_{\mathrm{m}}\right)$ values at other frequencies and other excitation amplitudes $\left(B_{\mathrm{NHCR}}=0.0175\right)$ that are not apparently related with low/high jumps of the membrane velocity RMS values, as shown for $B_{\mathrm{NHCR}} \leq B \leq B_{\mathrm{HCR}}$, corresponding to low membrane velocity amplitude $\left(v_{\mathrm{m}}<1.2 \mathrm{~m} / \mathrm{s}\right)$. This is something not noticed before. 
Let complete the results under (single) sinusoidal excitation, by examining the out-of-phase mode (Test 6, Fig. 5). Concerning the system response, as expected, the resonance peak around the out-of-phase mode (mode 3, $f_{3}=87.83 \mathrm{~Hz}$, in Table 1) is observed on the sound pressures responses within the pipes (Fig. 5(e,f)) at low excitation amplitudes.

Observations presented above for Test 1 also hold for Test 6, except for the three following observations: First, the low/high jumps of the membrane velocity $v_{\mathrm{m}}$ RMS values also appears at $A=A_{\mathrm{HCR}}$ but the excitation amplitude $A_{\mathrm{HCR}}=0.2275$ is higher than in Test 1 (see Fig. $5(\mathrm{~b})$ ). Note that the $A_{\mathrm{HCR}}$ value corresponds to the TET threshold $\left(E_{\mathrm{TET}}=0.2275\right)$ given by the model (see Section 2.3). Since mode 3 exhibits out-of-phase motion within the pipes, the coupling box holding the membrane NES is close to a pressure node of the mode: its interaction with the membrane is weak, which explains the higher values of $A_{\mathrm{HCR}}$ and $E_{\mathrm{TET}}$ compared to in-phase mode. Second, in terms of membrane NES efficiency, the pressure reduction (on $p_{1}$ and $p_{2}$ ) due to the non linearity is limited to $\approx 45 \%$ of the underlying linear system responses, a lower pressure reduction than in Test 1 (see Fig. 5(g,h)). And third, contrary to Test 1 , in Test $6, A_{\mathrm{HCR}}=A_{\mathrm{NHCR}}$, high NHCR appear on Fig. 5(b) only at the immediate vicinity of frequencies where $v_{m}$ is low $\left(v_{m} \approx 1 \mathrm{~m} / \mathrm{s}\right)$ but close to the low/high jumps of the membrane velocity RMS values.

\subsection{Test 2: two excitation frequencies with constant amplitude ratio}

The behavior of the system under two excitation frequencies was explored in Tests 2, 3 and 4 (see Table 2), with a scanning frequency $f^{B}$ around the inphase mode (mode $4, f_{4}=98.32 \mathrm{~Hz}$, in Table 1 ), and a constant excitation 
amplitude ratio $A / B$. The second excitation frequency $\left(f^{A}=88 . \mathrm{Hz}\right)$ is fixed close to the resonance frequency of the out-of-phase mode (mode 3, $f_{3}=87.83 \mathrm{~Hz}$, in Table 1). Test 2 is presented on Fig. 6 (Board 1) and Fig. 7 (Board 2). In this test the amplitude ratio $A / B=2$. Tests 3 and 4 lead to similar observations and are not shown.

\subsubsection{Comparison with Test 1}

In Test 2, the run exhibits sudden abrupt changes above the excitation level $\left(A_{\mathrm{HCR}}, B_{\mathrm{HCR}}\right)=(2 \times 0.04375,0.04375)$ corresponding to jumps between low $(\approx 1.2 \mathrm{~m} / \mathrm{s})$ and high $(\approx 3.2 \mathrm{~m} / \mathrm{s})$ RMS values of the membrane velocity (see Fig. 6(b)). This corresponds to the same state change as for the system than in Test 1 , but occurring at a lower excitation level $\left(B_{\mathrm{HCR}}=0.07865\right.$ for Test 1 ) of the scanning frequency $f^{B}$. Interesting also is that the RMS value of the command current $i_{\mathrm{LS}}(\approx 0.22)$ at the excitation level $\left(A_{\mathrm{HCR}}, B_{\mathrm{HCR}}\right)$ for Test 2 is lower than the RMS value of the command current $i_{\mathrm{LS}}(\approx 0.3)$ at the excitation level $B_{\mathrm{HCR}}$ for Test 1 . This is a clear influence of the addition of the second excitation term at $f^{A}$ with $A=2 B$ compared to Test 1.

Concerning the efficiency, the membrane NES is able to reduce simultaneously the sound pressure RMS in both pipes for excitation levels $B \geq B_{\mathrm{HCR}}$ (resulting as the excitation level $(2 B, B)$ ) for frequencies where $v_{m} \geq 3 \mathrm{~m} / \mathrm{s}$ on Fig. 6(b). As shown in Fig. 6(g,h) for $B \geq 0.0525$, the response within pipe 1 (resp. pipe 2) represents only 65\% (resp. 50\%) of the underlying linear system response. Compared to Test 1, its efficiency is smaller but operates on a wider frequency range, and values superior to 1. (amplification) are not observed.

Concerning the energy frequency conversion ratios, as for Test $1, \operatorname{HCR}\left(v_{\mathrm{m}}\right)$ 
and $\operatorname{NHCR}\left(v_{\mathrm{m}}\right)$ seem adapted to detect changes in the nonlinear system behavior. Frequencies with $\operatorname{HCR}\left(v_{\mathrm{m}}\right)>0.02$ on Fig. 6(d) coincides with frequencies exhibiting high RMS values of $v_{m}$ on Fig. 6(b). And also, high $\operatorname{NHCR}\left(v_{\mathrm{m}}\right)$ on Fig. 6(c) appear at the immediate vicinity of frequencies where $v_{m}$ is low $\left(v_{m} \approx 1 \mathrm{~m} / \mathrm{s}\right)$, close to the low $/$ high jumps of the membrane velocity RMS values on Fig. 6(b). This scenario has been observed numerically for a similar system in [9]. But contrary to Test $1, \operatorname{HCR}\left(v_{\mathrm{m}}\right)$ now can easily exceed $10 \%$, and at some frequencies high values for $\operatorname{HCR}\left(v_{\mathrm{m}}\right)$ can be observed simultaneously to high $\operatorname{NHCR}\left(v_{\mathrm{m}}\right)$. It indicates that solutions approximated by limiting the harmonics terms to the fundamental frequencies for the membrane NES may hardly describe all the nonlinear effects of the membrane with two excitation frequencies.

Finally we observe that $\left(A_{\mathrm{NHCR}}, B_{\mathrm{NHCR}}\right)=(2 \times 0.03065,0.03065)$ showing that Test 2 also exhibit high $\operatorname{NHCR}\left(v_{\mathrm{m}}\right)$ values at other frequencies and other excitation levels that are not apparently related with low/high jumps of the membrane velocity RMS values, as shown for $B_{\mathrm{NHCR}} \leq B \leq B_{\mathrm{HCR}}$. These high NHCR values appear for low membrane velocity amplitude $\left(v_{\mathrm{m}}<\right.$ $1.2 \mathrm{~m} / \mathrm{s})$.

\subsubsection{Membrane NES effects at the two resonance frequencies}

Now a deeper understanding of how the in-phase mode and the out-ofphase mode are influenced by the membrane NES is proposed. The in-phase mode and the out-of-phase mode, which both contribute to pressure in pipes 1 and 2, are separated thanks to Fourier magnitude ratios at excitation frequencies. The Fourier magnitude ratios $P_{1}\left(f^{B}\right) / I_{\mathrm{LS}}\left(f^{B}\right)$ and $P_{2}\left(f^{B}\right) / I_{\mathrm{LS}}\left(f^{B}\right)$ shown Fig. 7(b,d) are related to the amplitude of the in-phase mode whereas 
the Fourier magnitude ratios $P_{1}\left(f^{A}\right) / I_{\mathrm{LS}}\left(f^{A}\right)$ and $P_{2}\left(f^{A}\right) / I_{\mathrm{LS}}\left(f^{A}\right)$ shown Fig. $7(\mathrm{a}, \mathrm{c})$ are related to the amplitude of the out-of-phase mode. The four ratios decrease when the excitation level increases: the NES acts simultaneously and efficiently at both excitation frequencies, on both modes. But the amplitude of the out-of-phase mode is not really sensitive to $f^{B}$ : the run response do not vary much along $f^{B}$ (see Figs. $7(\mathrm{a}, \mathrm{c})$ ), whereas the amplitude of the in-phase mode (see Figs. $7(\mathrm{~b}, \mathrm{~d})$ ) varies with $f^{B}$ similarly to the response RMS of pipe 2 (see Fig. 6(f)).

A symmetrical situation is observed for Test 8 (see Fig. 8, Board 2), for which the excitation frequency $f^{A}$ varies while $f^{B}$ is now constant $(98.8 \mathrm{~Hz})$. As for Test 2, the four ratios decrease when the excitation level increases: the NES acts simultaneously and efficiently at both excitation frequencies. But contrary to Test 2, the in-phase mode 4 is now less dependent upon $f^{A}$ (Figs. 8(a,c)), and the out-of-phase mode varies with $f^{A}$. Even if with $\operatorname{HCR}\left(v_{\mathrm{m}}\right)$ and $\operatorname{NHCR}\left(v_{\mathrm{m}}\right)$ values up to $70 \%$, most of the signal energy can be away from $f^{A}$ and $f^{B}$, a single NES can reduce simultaneously the response of 2 modes excited at their respective resonance frequencies. This scenario is interpreted as a simultaneous control of two one-to-one resonances by a single NES, which was first considered numerically in [8].

\subsection{Test 5: two-frequency test with one amplitude constant}

Test 5 was designed essentially to analyze the influence of a second additive periodic excitation at a different frequency. It was especially used to test the efficiency for sound attenuation. Test 5 starts from periodic excitation defined with $A=0, B=0.0656$ and $f^{B}$ scanned in the range $[94,104] \mathrm{Hz}$ as in Test 1 . $B=0.0656$ has been chosen so that the system is just below the 
excitation level $B_{\mathrm{HCR}}\left(B_{\mathrm{HCR}}=0.07875\right.$ for Test 1$)$. Next a second additive periodic excitation is added at the frequency $f^{A}=88$. Hz (near the out-ofphase mode) and several excitation levels were considered. The results of Test 5 are summarized in Fig. 9 following Board 1 representation and Fig. 10 following Board 2 representation.

\subsubsection{Noise reduction by an additive periodic excitation.}

Noise reduction can be analyzed observing the efficiency RMS ratios shown Fig. 9(g,h). Increasing $A$, the efficiency RMS ratios decreases at the same time, in all frequency band and almost with the same magnitude. The responses $\left(p_{1}\right.$ and $\left.p_{2}\right)$ are as low as $50 \%$ of what should have been expected if the system was linear.

A nice effect is observed on Fig. 9(f): an increase in $A$ not only diminishes the relative (with respect to a underlying linear system) response in pipe 2, but it also diminishes the response RMS in pipe 2, with up to $40 \%$ improvement in a narrow band around the resonance frequency of the in-phase mode. In parallel the response RMS in pipe 1 (see Fig. 9(e)), always increases varying (versus $f^{B}$ ) greatly at low excitation level $(A$ low) and being constant at high excitation level. At low excitation level ( $A$ low), we see the response at $f^{B}$, at high excitation level ( $A$ high) the response at $f^{A}$ becomes dominant.

The last point is to check if the noise reduction is due to simultaneous control of the two modes by the NES. The response can be obtained from Fig. 10. We observe that the four ratios $P_{1}\left(f^{A}\right) / I_{\mathrm{LS}}\left(f^{A}\right), P_{2}\left(f^{A}\right) / I_{\mathrm{LS}}\left(f^{A}\right)$, $P_{1}\left(f^{B}\right) / I_{\mathrm{LS}}\left(f^{B}\right)$ and $P_{2}\left(f^{B}\right) / I_{\mathrm{LS}}\left(f^{B}\right)$ decrease when the excitation level increases showing that the NES acts simultaneously and efficiently on both excitation frequencies. 
Finally, the presence of the term in $f^{A}$ in the source decreases the response at $f^{B}$, and the response RMS in the tube 2 . We point out that this is a reduction of absolute quantities. This could be useful in view of applications: in such a system, contrary to intuition, the energy at an annoying frequency can be dimmed by adding a perturbation at another frequency. In other terms, noise reduction can be controlled by addition of noise.

A similar scenario was first proposed and studied numerically in [10] considering a single NES attached to a 1-DOF system but with frequencies close to each other.

\subsubsection{Energy frequency conversion}

Energy frequency conversions happen in Test 5 for all values of $A$. In Fig. $9(\mathrm{c}, \mathrm{d})$, as $A$ increases, $\operatorname{HCR}\left(v_{\mathrm{m}}\right)$ and $\operatorname{NHCR}\left(v_{\mathrm{m}}\right)$ increase, up to $30 \%$ for $\operatorname{HCR}\left(v_{\mathrm{m}}\right)$ and up to $75 \%$ for $\operatorname{NHCR}\left(v_{\mathrm{m}}\right)$.

At high excitation level, the size of the harmonic conversion zone increases with the excitation level and the harmonic conversion zones coexist (simultaneously) with the nonharmonic conversion zones.

The interesting point is that it seems that the system is not very sensitive to detuning and that there is a continuity of behavior with respect to the variables $A$. The transition from low to high membrane velocity RMS values is here smooth without jumps (see Fig. 9(b)).

\subsection{Frequency conversion in the two excitation amplitudes plane}

We observed in the previous sections that an addition of a detuned excitation component to an existing excitation signal tends to trigger the high amplitude response regime. In this section an overview of the frequency 
conversion in the two excitation amplitudes plane is proposed.

The excitation levels used in Tests 1 to 9 are plotted in $(A, B)$-plane in Fig. 11. A point corresponds to a run of the test at the corresponding excitation level $(A, B)$. A circle marker is used instead of a point if during the run, at least one experiment (at one frequency of the scan) gives $\operatorname{HCR}\left(v_{\mathrm{m}}\right) \geq 0.02$. Similarly, a star marker is used when $\operatorname{NHCR}\left(v_{\mathrm{m}}\right) \geq 0.02$. A big marker indicates the HCR threshold $\left(A_{\mathrm{HCR}}, B_{\mathrm{HCR}}\right)$ and the NHCR threshold $\left.\left(A_{\mathrm{NHCR}}, B_{\mathrm{NHCR}}\right)\right)$ occurrence of the given marker for each test. Note that when a circle marker and a star marker appear simultaneously this do not imply that $\operatorname{HCR}\left(v_{\mathrm{m}}\right) \geq 0.02$ and $\operatorname{NHCR}\left(v_{\mathrm{m}}\right) \geq 0.02$ occur at the same frequency (i.e. in the same experiment). Fig. 11(a) sums up tests with $f^{A}=88 \mathrm{~Hz}$ (fixed) and $f^{B}$ in the $[94 ., 104$.] Hz range (Tests 1 to 5) whereas Fig. 11(b) sums up tests with $f^{B}=98.8 \mathrm{~Hz}$ and $f^{A}$ in [85., 95.] Hz (Tests 6 to 9$)$.

Except Test 1 (Fig. 11(a), vertical segment line), the bigger star and circle markers are close or equal, showing that energy harmonic and non-harmonic frequency conversions start at the same excitation level. Moreover except Test 4 (Fig. 11(a), smaller slope segment line), circle and star markers appear simultaneously for excitation levels greater than $\left(A_{\mathrm{HCR}}, B_{\mathrm{HCR}}\right)$. Higher excitation level can be understood considering one or two excitation components depending on the tests. It can also be understood as the higher excitation RMS for a given test at which stars or circles appear.

It is reasonable from Fig.11(a) that bigger circles could be ascribed, in a first attempt, to the segment line defined by the two points $\left(0, B_{\mathrm{HCR}}^{1}\right)$ and $\left(A_{\mathrm{HCR}}^{6}, 0\right)$ where $B_{\mathrm{HCR}}^{1} \approx 0.07875$ denotes the $B_{\mathrm{HCR}}$ value associated to Test 
1 and $A_{\mathrm{HCR}}^{6} \approx 0.2275$ denotes $A_{\mathrm{HCR}}$ value associated to Test 6 . The same observations hold for Fig.11(b), may be with a slighter dispersion around the segment line $\left(0, B_{\mathrm{HCR}}^{1}\right)\left(A_{\mathrm{HCR}}^{6}, 0\right)$. This difference could come from the imperfection of the localization of the actual resonance frequencies in the tests. The meaning of resonance frequency must be clarified, because the system state often varies along a run. By resonance frequency we mean here the frequency at which the responses peak.

It is unclear whether the $\left(A_{\mathrm{HCR}}, B_{\mathrm{HCR}}\right)$ relationship is linear as the segment suggests, which would be consistent with a regime change driven by the amplitude of the excitation, or if the relationship is quadratic, which would mean a regime change driven by an energetic indicator. In case of sinusoidal excitation, theoretical results show that the regime change is driven by the amplitude (see for example [2]). In case of multi-frequency excitation, limited theoretical results show that for a given amplitude, the regime change is driven by the other amplitude [10]. The linear relationship has to be confirmed. If it is realistic, it would be interesting to study the effects of spikes in the source and response signals in order to induce the high amplitude regime with the least possible energy.

\section{Conclusion}

We presented an experimental study in the acoustic domain of a two degree-of-freedom linear system coupled to a single NES under two-frequency excitations. The set-up used consisted of two pipes with different lengths, a visco-elastic membrane (NES) and its coupling box excited by an acoustic source. Indicators based on response RMS and Fourier transform were built 
for three purposes: (i) analyzing the efficiency of the NES as a noise absorber, (ii) detecting the nonlinear effects in terms of energy frequency conversion from the excitation frequencies towards their inter modulation frequencies (HCR) and towards frequencies different from the excitation frequencies and any of their possible inter modulation frequencies (NHCR), and (iii) separating the resonant motion components. These indicators can be applied for periodic and quasiperiodic excitations.

The system behavior was first investigated under periodic excitation validating the set-up design, showing that the membrane acts as a NES for noise reduction around the two resonance frequencies of the acoustic medium, and validating the indicators. Next, excitations with two frequencies were investigated using the excitation frequencies and the excitation amplitudes as control parameters. The main findings of this study are:

- The HCR threshold is well adapted to detect low/high jumps of the membrane velocity RMS values characterizing the excitation level where the NES is efficient.

- Simultaneous reduction of amplitudes for two-DOF by a single NES is observed experimentally. This is a counter-intuitive feature that extends the application range of NES. In particular a simultaneous control of two one-to-one resonances by the NES is observed.

- An absolute reduction of the amplitude of vibration in a two-DOF linear system coupled to a single NES can be triggered by an addition of a significantly off-tuned perturbation to an initial harmonic source. This result, again counter-intuitive, delivers a new technique for pas- 
sive reduction of vibrations. In all cases, the introduction of energy at a second excitation frequency appears favorable to lower frequency conversion threshold and lower noise within the system.

Finally, an overview of the frequency conversion in the two excitation amplitudes plane, yields to an observation not yet established in theory: the frontier (in the excitation amplitudes plane) that characterizes the change of states of the system (detection of low/high jumps) may be controlled by a linear combination of the HCR thresholds. This opens new perspectives for theoretical and numerical studies of nonlinear systems.

\section{References}

[1] A. Vakakis, O. Gendelman, L. Bergman, D. McFarland, G. Kerschen, Y. Lee, Nonlinear targeted energy transfer in mechanical and structural systems, Vol. 156 of Solid mechanics and its applications, Springer, 2008.

[2] Y. Starosvetsky, O. V. Gendelman, Attractors of harmonically forced linear oscillator with attached nonlinear energy sink. ii: Optimization of a nonlinear vibration absorber, Nonlinear Dynamics 51 (2008) 47-57.

[3] O. V. Gendelman, E. Gourdon, C.H. Lamarque, Quasiperiodic energy pumping in coupled oscillators under periodic forcing, Journal of Sound and Vibration 294 (2006) 651-662.

[4] Y. Starosvetsky, O. Gendelman, Dynamics of a strongly nonlinear vibration absorber coupled to a harmonically excited two-degree-of-freedom system, Journal of Sound and Vibration 312 (2008) 234-256. 
[5] R. Bellet, B. Cochelin, P. Herzog, P.-O. Mattei, Experimental study of targeted energy transfer from an acoustic system to a nonlinear membrane absorber, Journal of Sound and Vibration 329 (2010) 2768-2791.

[6] R. Bellet, B. Cochelin, R. Côte, P.-O. Mattei, Enhancing the dynamic range of targeted energy transfer in acoustics using several nonlinear membrane absorbers, Journal of Sound and Vibration 331 (26) (2012) $5657-5668$.

[7] T. Pham, S. Pernot, C. Lamarque, Competitive energy transfer between a two degree-of-freedom dynamic system and an absorber with essential nonlinearity, Nonlinear Dynamics 62 (2010) 573-592.

[8] T. Pham, C. Lamarque, A. T. Savadkoohi, Multi-resonance capturing in a two-degree-of-freedom system under two different harmonic excitations, Journal of Vibration and Control 18 (3) (2012) 451-466.

[9] S. Bellizzi, R. Côte, M. Pachebat, Responses of a two degree-of-freedom system coupled to a nonlinear damper under multi-forcing frequencies, Journal of Sound and Vibration 332 (2013) 1639-1653.

[10] Y. Starosvetsky, O. Gendelman, Response regimes in forced system with non-linear energy sink: quasi-periodic and random forcing, Nonlinear Dynamics 64 (2011) 177-195.

[11] R. Mariani, S. Bellizzi, B. Cochelin, P. Herzog, P.-O. Mattei, Toward an adjustable nonlinear low frequency acoustic absorber, Journal of Sound and Vibration 330 (2011) 5245-5258. 
[12] S. Bellizzi, B. Cochelin, P. Herzog, P. Mattei, C. Pinhéde, Experimental investigation of low frequency noise reduction using a nonlinear vibracoustic absorber, in: Proceedings of the ASME International Design Engineering Technical Conference \& Computers and Information in Engineering Conference (DETC2011)-Volume 1, Parts A \& B, 2011.

[13] M. Rossi, Acoustics and Electroacoustics, 1st Edition, Artech House Inc., Norwood (USA), 1988.

[14] ManLab, Version 2.0, (http://manlab.lma.cnrs-mrs.fr/), 2010.

[15] B. Cochelin, C. Vergez, A high order purely frequency-based harmonic balance formulation for continuation of periodic solutions, Journal of Sound and Vibration 324 (2009) 243-262.

[16] B. Cochelin, N. Damil, E. Allgowe, Méthode asymptotique numérique, Hermes Lavoisier, 2007.

[17] J. Shao, B. Cochelin, Passive control of resonances by nonlinear absorbers, in: MATEC Web of Conferences, CSNDD 2012 - International Conference on Structural Nonlinear Dynamics and Diagnosis, Vol. 1, 2012.

[18] G. Gómez, J.-M. Mondelo, C. Simó, A collocation method for the numerical fourier analysis of quasi-periodic functions. i: Numerical tests and examples, Discrete and Continuous Dynamical Systems - Series B 14 (2010) 41-74. 
[19] O. Gendelman, Y. Starosvetsky, Quasi-periodic response regimes of linear oscillator coupled to nonlinear energy sink under periodic forcing, Journal of Applied Mechanics 74 (2007) 325-331.

\section{List of Figures}

1 (a) Picture and (b-c) schemas of the set-up. . . . . . . . 5

$2 \quad \mathrm{FRF}$ (a) $p_{1} / i_{\mathrm{LS}}$ (b) $p_{2} / i_{\mathrm{LS}}$, system without NES. Comparison experiment (dotted lines) and model (continuous line). . . . . 11

3 RMS responses (a) $p_{1}$, (b) $p_{2}$ and (c) $v_{\mathrm{m}}$, system with NES. Comparison of experiment (dotted lines) and model (continuous lines) for several excitation levels: $E=0.004375 \mathrm{~V}$ (yellow curves), $E=0.07 \mathrm{~V}$ (blue curves), $E=0.07875 \mathrm{~V}$ (red curves), $E=0.1312 \mathrm{~V}$ (green curves) and $E=0.2275 \mathrm{~V}$ (magenta curves); also shown: Nonlinear Normal Modes (black curves). . . . . . . . . . . . . . . . . 12

4 Test 1, Board 1: $A=0$ and $B=0.004375,0.0175,0.030625$, $0.04375,0.0525,0.06125,0.07875,0.091875,0.1028125,0.1575$, $0.2625,0.35$ (from blue to red color). $\left(A_{\mathrm{HCR}}, B_{\mathrm{HCR}}\right)=(0,0.07875)$ and $\left(A_{\mathrm{NHCR}}, B_{\mathrm{NHCR}}\right)=(0,0.0175) . \ldots \ldots \ldots . \ldots . \ldots 42$

$5 \quad$ Test 6, Board 1: $A=0.013125,0.06125,0.105,0.14,0.175$, $0.1925,0.21,0.2275,0.245,0.2625,0.2975,0.35$ (from blue to red color $)$ and $B=0 .\left(A_{\mathrm{HCR}}, B_{\mathrm{HCR}}\right)=\left(A_{\mathrm{NHCR}}, B_{\mathrm{NHCR}}\right)=$ $(0.2275,0)$. 
Test 2, Board 1: $A=2 B, B=0.00875,0.0175,0.02625$, $0.03065,0.035,0.0294,0.04375,0.0525,0.06125,0.07,0.07875,0.0875$ (from blue to red color); $f^{A}=88 . \mathrm{Hz}, f^{B}=[94,104.] \mathrm{Hz}$. $\left(A_{\mathrm{HCR}}, B_{\mathrm{HCR}}\right)=\left(2 B_{\mathrm{HCR}}, 0.04375\right)$ and $\left(A_{\mathrm{NHCR}}, B_{\mathrm{NHCR}}\right)=\left(2 B_{\mathrm{NHCR}}, 0.03065\right) .44$

7 Test 2, Board 2: $A=2 B, B=0.00875,0.0175,0.02625$, $0.03065,0.035,0.0294,0.04375,0.0525,0.06125,0.07,0.07875,0.0875$ (from blue to red color); $f^{A}=88 . \mathrm{Hz}, f^{B}=[94,104.] \mathrm{Hz}$. $\left(A_{\mathrm{HCR}}, B_{\mathrm{HCR}}\right)=\left(2 B_{\mathrm{HCR}}, 0.04375\right)$ and $\left(A_{\mathrm{NHCR}}, B_{\mathrm{NHCR}}\right)=\left(2 B_{\mathrm{NHCR}}, 0.03065\right) .45$

8 Test 8, Board 2: $A=0.035,0.06565,0.1006,0.1161,0.14$, $0.1575,0.1662,0.1861,0.2144,0.245,0.2931,0.35$ and $B=$ $A / 8$ (from blue to red color); $f^{A}=[85,95.] \mathrm{Hz}, f^{B}=98.8 \mathrm{~Hz}$. $\left(A_{\mathrm{HCR}}, B_{\mathrm{HCR}}\right)=\left(0.1662, A_{\mathrm{HCR}} / 8\right)$ and $\left(A_{\mathrm{NHCR}}, B_{\mathrm{NHCR}}\right)=\left(0.1575, A_{\mathrm{NHCR}} / 8\right) .46$

$9 \quad$ Test 5, Board 1: $B=0.0656 ; A=0.00438,0.00875,0.0175$, 0.0263, 0.0350, 0.0481, 0.0569, 0.0700, 0.0744, 0.0788, 0.0831, 0.0875 (from blue to red color); $f^{A}=88 . \mathrm{Hz}, f^{B}=[94 ., 104.] \mathrm{Hz}$. $\left(A_{\mathrm{HCR}}, B_{\mathrm{HCR}}\right)=(0.0175,0.0656)$ and $\left(A_{\mathrm{NHCR}}, B_{\mathrm{NHCR}}\right)=(0.00438,0.0656) .47$

10 Test 5, Board 2: $B=0.0656 ; A=0.00438,0.00875,0.0175$, 0.0263, 0.0350, 0.0481, 0.0569, 0.0700, 0.0744, 0.0788, 0.0831, 0.0875 (from blue to red color); $f^{A}=88 . \mathrm{Hz}, f^{B}=[94 ., 104.] \mathrm{Hz}$. $\left(A_{\mathrm{HCR}}, B_{\mathrm{HCR}}\right)=(0.0175,0.0656)$ and $\left(A_{\mathrm{NHCR}}, B_{\mathrm{NHCR}}\right)=(0.00438,0.0656) .48$

11 The $\left\{\operatorname{HCR}\left(v_{\mathrm{m}}\right) \geq 0.02\right\}$ (circle markers) and $\left\{\operatorname{NHCR}\left(v_{\mathrm{m}}\right) \geq\right.$ $0.02\}$ (star markers) zones in $(A, B)$-plane for (a) Tests 1 to 5 and (b) Tests 6 to 9. Big star markers correspond to HCR threshold $\left(A_{\mathrm{HCR}}, B_{\mathrm{HCR}}\right)$, big circle markers correspond

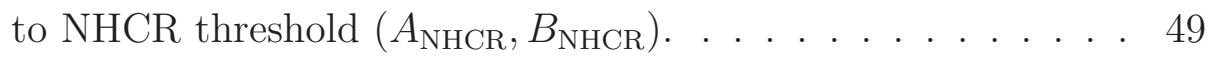




\section{List of Tables}

1 Resonance frequencies and mode shapes of the undamped underlying linear model with membrane NES. . . . . . . . . . . . 13

2 Excitation parameters for the tests. . . . . . . . . . 17 

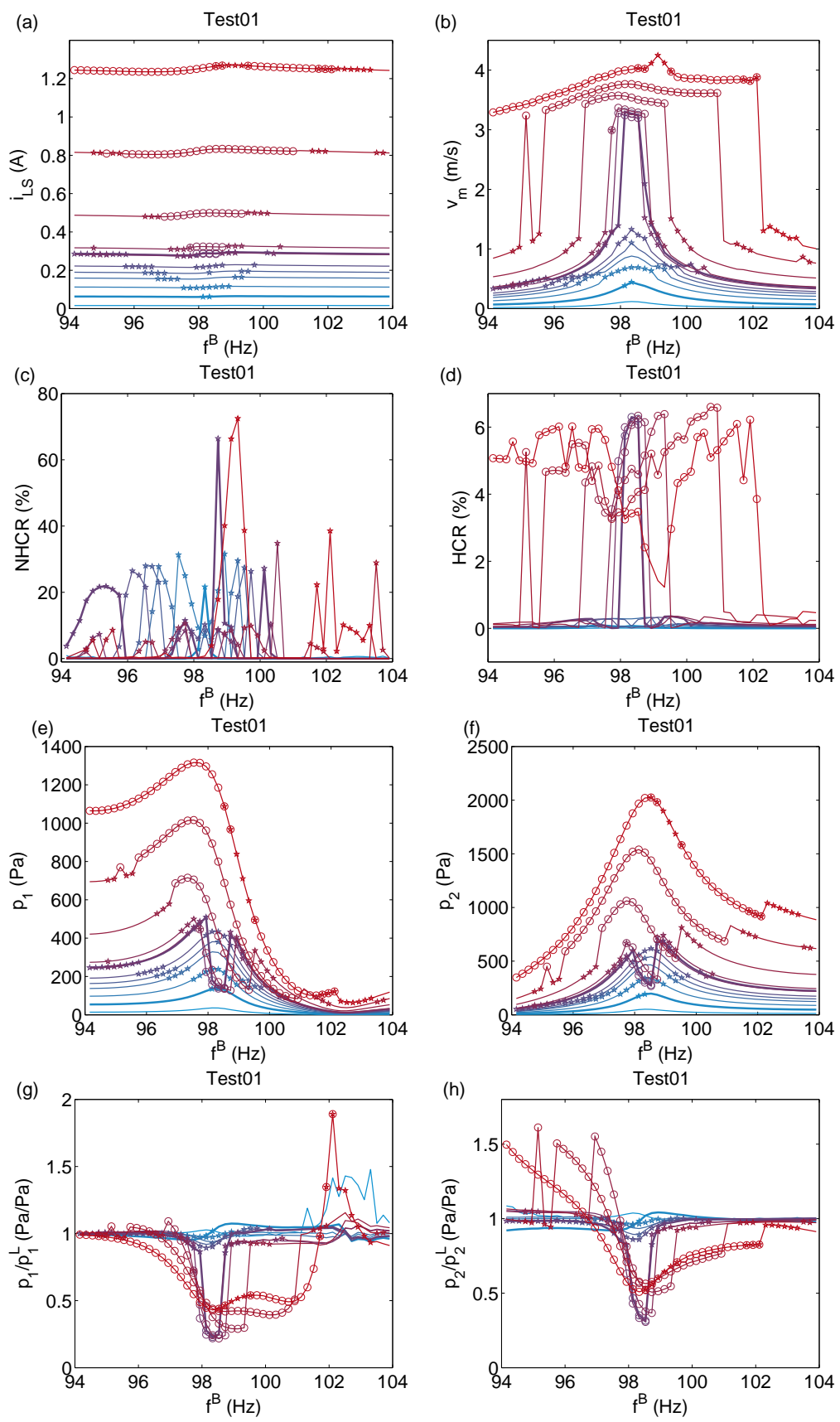

Figure 4: Test 1, Board 1: $A=0$ and $B=0.004375,0.0175,0.030625,0.04375,0.0525$, $0.06125,0.07875,0.091875,0.1028125,0.1575,0.2625,0.35$ (from blue to red color). $\left(A_{\mathrm{HCR}}, B_{\mathrm{HCR}}\right)=(0,0.07875)$ and $\left(A_{\mathrm{NHCR}}, B_{\mathrm{NHCR}}\right)=(0,0.0175)$. 

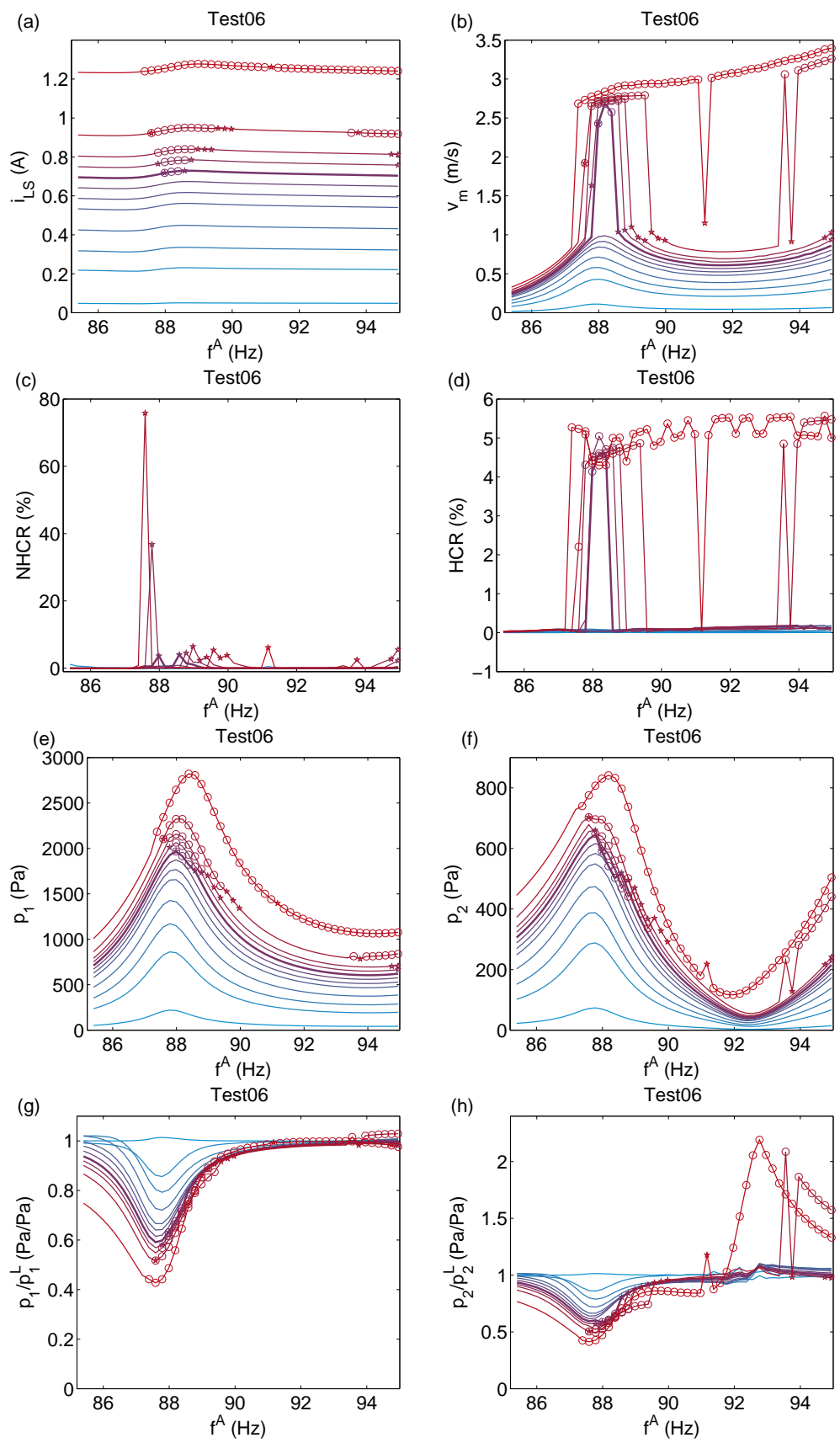

Figure 5: Test 6, Board 1: $A=0.013125,0.06125,0.105,0.14,0.175,0.1925,0.21$, $0.2275,0.245,0.2625,0.2975,0.35$ (from blue to red color) and $B=0 .\left(A_{\mathrm{HCR}}, B_{\mathrm{HCR}}\right)=$ $\left(A_{\mathrm{NHCR}}, B_{\mathrm{NHCR}}\right)=(0.2275,0)$. 

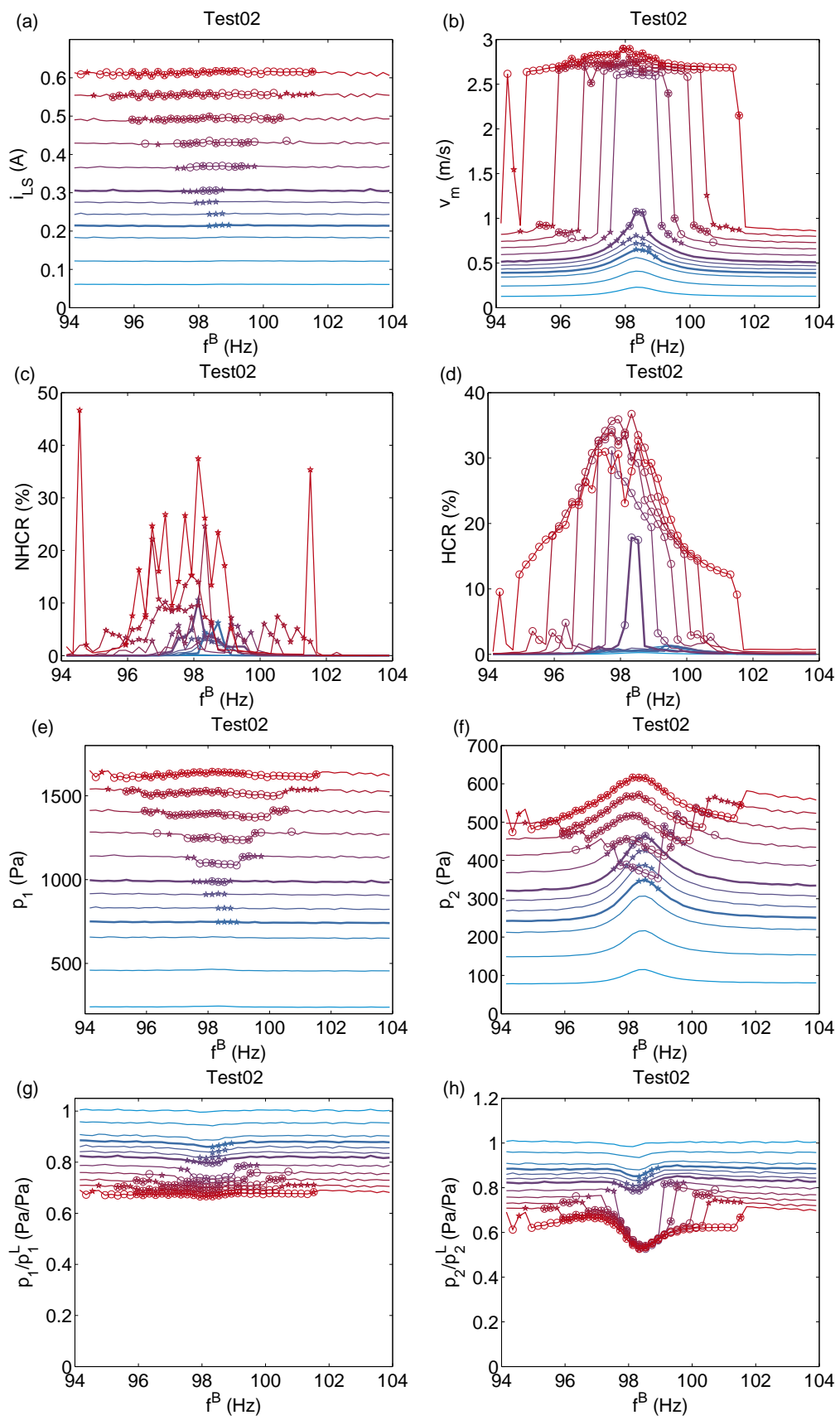

Figure 6: Test 2, Board 1: $A=2 B, B=0.00875,0.0175,0.02625,0.03065,0.035$, 0.0294, 0.04375, 0.0525, 0.06125, 0.07, 0.07875, 0.0875 (from blue to red color); $f^{A}=$ 88. Hz, $f^{B}=[94,104.] \mathrm{Hz} .\left(A_{\mathrm{HCR}}, B_{\mathrm{HCR}}\right)=\left(2 B_{\mathrm{HCR}}, 0.04375\right)$ and $\left(A_{\mathrm{NHCR}}, B_{\mathrm{NHCR}}\right)=$ $\left(2 B_{\mathrm{NHCR}}, 0.03065\right)$. 

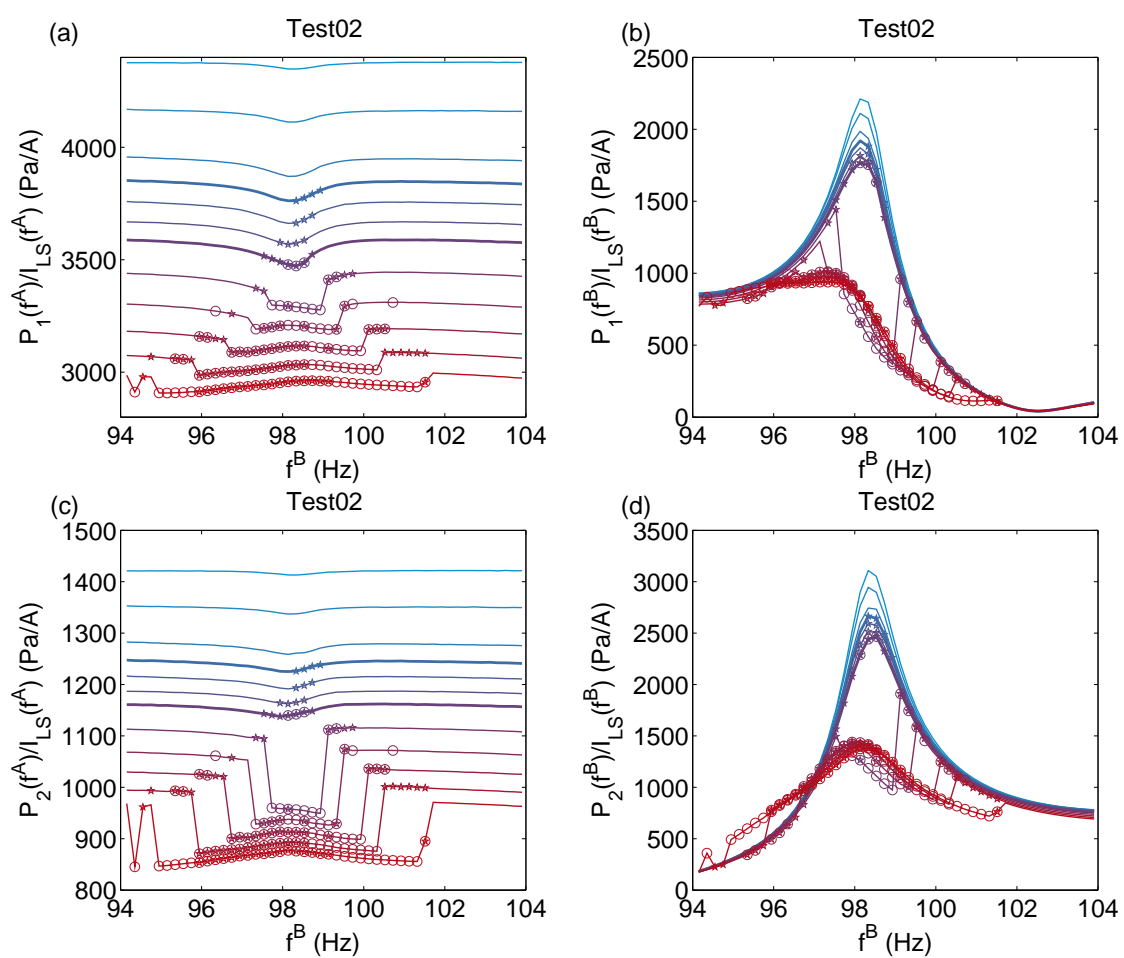

Figure 7: Test 2, Board 2: $A=2 B, B=0.00875,0.0175,0.02625,0.03065,0.035$, $0.0294,0.04375,0.0525,0.06125,0.07,0.07875,0.0875$ (from blue to red color); $f^{A}=$ 88. $\mathrm{Hz}, f^{B}=[94,104.] \mathrm{Hz} .\left(A_{\mathrm{HCR}}, B_{\mathrm{HCR}}\right)=\left(2 B_{\mathrm{HCR}}, 0.04375\right)$ and $\left(A_{\mathrm{NHCR}}, B_{\mathrm{NHCR}}\right)=$ $\left(2 B_{\mathrm{NHCR}}, 0.03065\right)$. 

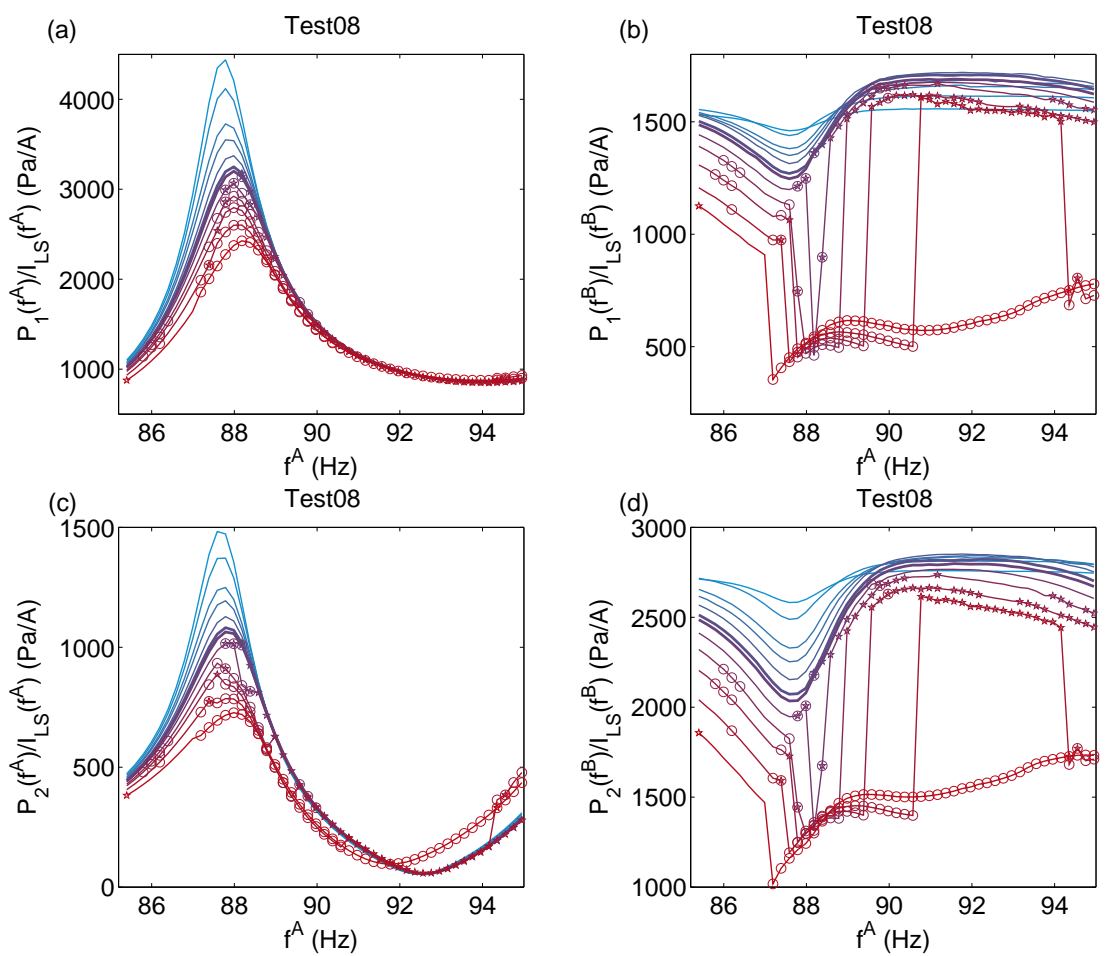

Figure 8: Test 8, Board 2: $A=0.035,0.06565,0.1006,0.1161,0.14,0.1575,0.1662$, $0.1861,0.2144,0.245,0.2931,0.35$ and $B=A / 8$ (from blue to red color); $f^{A}=$ $[85,95.] \mathrm{Hz}, f^{B}=98.8 \mathrm{~Hz} .\left(A_{\mathrm{HCR}}, B_{\mathrm{HCR}}\right)=\left(0.1662, A_{\mathrm{HCR}} / 8\right)$ and $\left(A_{\mathrm{NHCR}}, B_{\mathrm{NHCR}}\right)=$ $\left(0.1575, A_{\mathrm{NHCR}} / 8\right)$. 

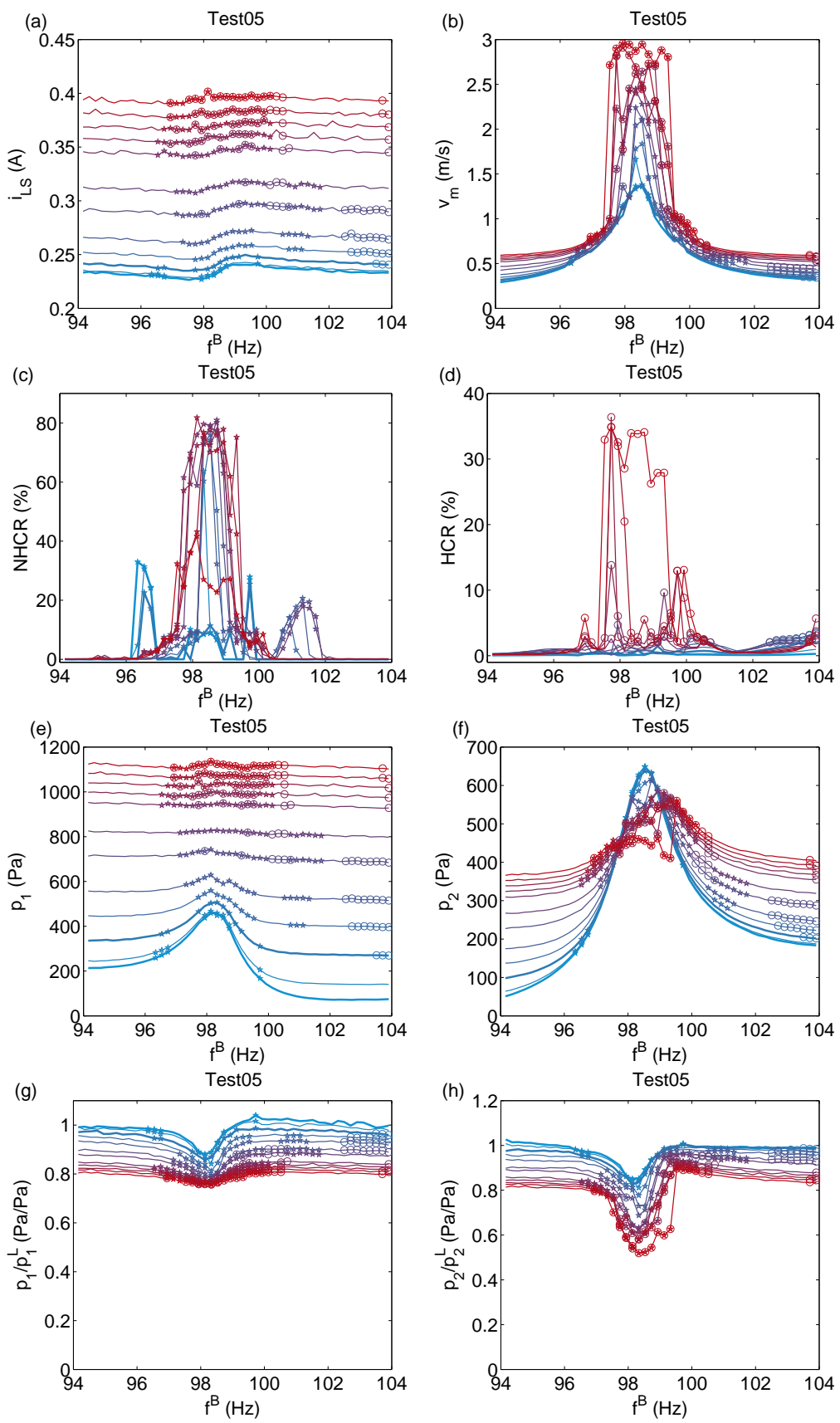

Figure 9: Test 5, Board 1: $B=0.0656 ; A=0.00438,0.00875,0.0175,0.0263,0.0350$, 0.0481, 0.0569, 0.0700, 0.0744, 0.0788, 0.0831, 0.0875 (from blue to red color); $f^{A}=$ 88. Hz, $f^{B}=[94 ., 104.] \mathrm{Hz} .\left(A_{\mathrm{HCR}}, B_{\mathrm{HCR}}\right)=(0.0175,0.0656)$ and $\left(A_{\mathrm{NHCR}}, B_{\mathrm{NHCR}}\right)=$ $(0.00438,0.0656)$. 

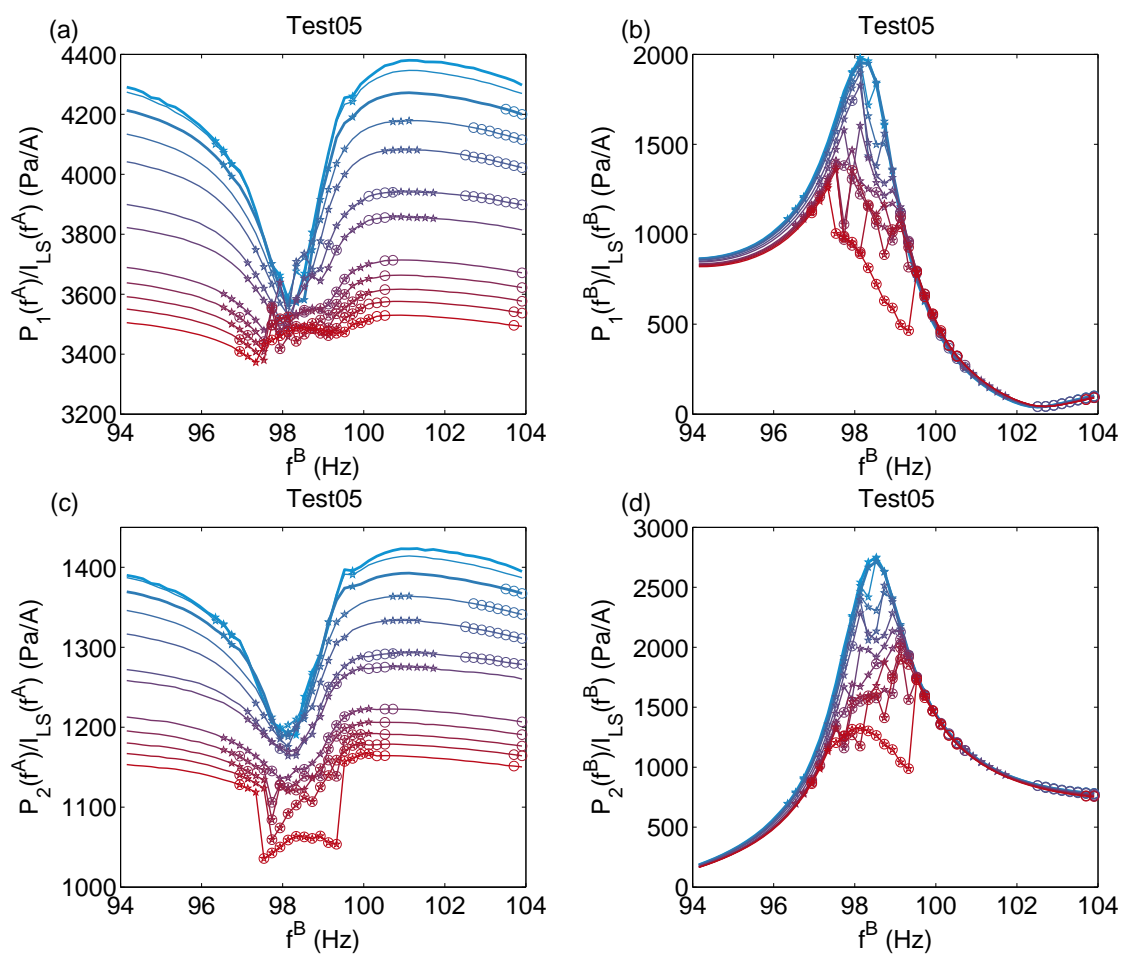

Figure 10: Test 5, Board 2: $B=0.0656 ; A=0.00438,0.00875,0.0175,0.0263,0.0350$, $0.0481,0.0569,0.0700,0.0744,0.0788,0.0831,0.0875$ (from blue to red color); $f^{A}=$ 88. $\mathrm{Hz}, f^{B}=[94 ., 104.] \mathrm{Hz} .\left(A_{\mathrm{HCR}}, B_{\mathrm{HCR}}\right)=(0.0175,0.0656)$ and $\left(A_{\mathrm{NHCR}}, B_{\mathrm{NHCR}}\right)=$ $(0.00438,0.0656)$. 

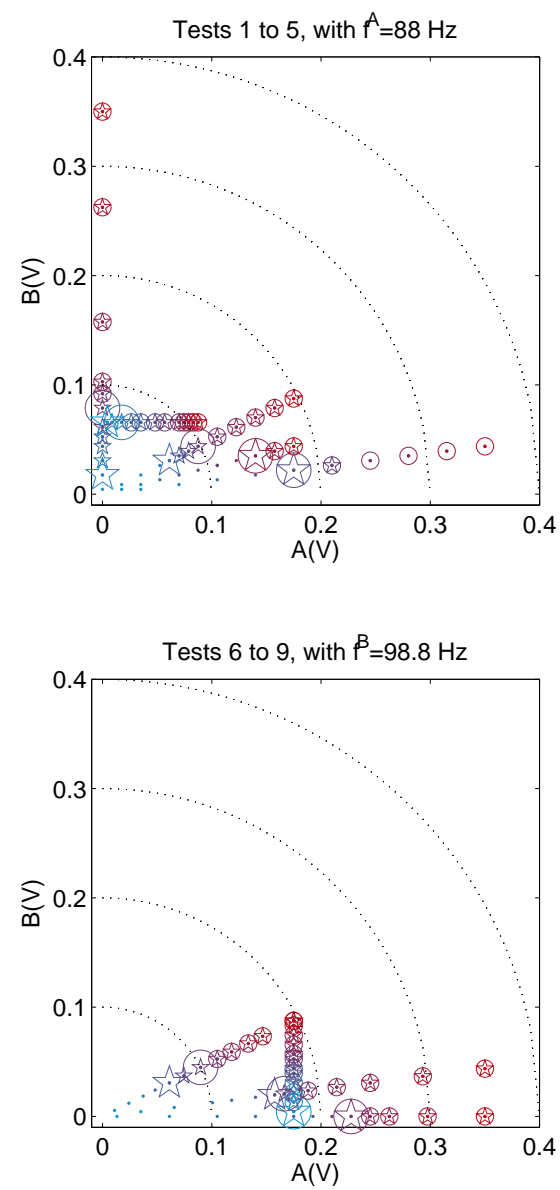

Figure 11: The $\left\{\operatorname{HCR}\left(v_{\mathrm{m}}\right) \geq 0.02\right\}$ (circle markers) and $\left\{\operatorname{NHCR}\left(v_{\mathrm{m}}\right) \geq 0.02\right\}$ (star markers) zones in $(A, B)$-plane for (a) Tests 1 to 5 and (b) Tests 6 to 9. Big star markers correspond to $\mathrm{HCR}$ threshold $\left(A_{\mathrm{HCR}}, B_{\mathrm{HCR}}\right)$, big circle markers correspond to NHCR threshold $\left(A_{\mathrm{NHCR}}, B_{\mathrm{NHCR}}\right)$. 